\title{
A Class of Fuzzy Variational Inequality Based on Monotonicity of Fuzzy Mappings
}

\author{
Zezhong $W u^{1}$ and Jiuping $X u^{2}$ \\ ${ }^{1}$ College of Mathematics, Chengdu University of Information Technology, Chengdu 610225, China \\ ${ }^{2}$ Uncertainty Decision-Making Laboratory, School of Business and Administration, Sichuan University, Chengdu 610064, China \\ Correspondence should be addressed to Jiuping Xu; xujiuping@scu.edu.cn
}

Received 11 May 2013; Accepted 1 September 2013

Academic Editor: Kanishka Perera

Copyright (c) $2013 \mathrm{Z}$. Wu and J. Xu. This is an open access article distributed under the Creative Commons Attribution License, which permits unrestricted use, distribution, and reproduction in any medium, provided the original work is properly cited.

\begin{abstract}
Invex monotonicity and pseudoinvex monotonicity of fuzzy mappings are introduced in this paper, and relations are discussed between invex monotonicity (pseudoinvex monotonicity) and invexity (pseudoinvexity) of fuzzy mappings. The existence of a solution to the fuzzy variational-like inequality is discussed, and the existence theorem can be achieved. Furthermore, some extended properties of the fuzzy variational-like inequality are researched. Finally, method of solution is discussed based on genetic algorithm.
\end{abstract}

\section{Introduction}

In [1], Chang and Zadeh introduced the concept of fuzzy mapping. Since then, fuzzy mapping has been extensively studied by many authors. Nanda and Kar [2] proposed a concept of convex fuzzy mapping in 1992 and proved that a fuzzy mapping is convex if and only if its epigraph is a convex set. In recent years, there have been increasing attempts to weaken the convexity condition of fuzzy mapping, such as Yan and $\mathrm{Xu}$ [3], Panigrahi et al. [4], and $\mathrm{Wu}$ and $\mathrm{Xu}[5,6]$. It is well known that in classical (non-fuzzy) convex analysis, some properties are shared by different kinds of functions that are more general than convex functions, which has given rise to the study of generalized convexity and later to that of generalized monotonicity. Just as convex functions are characterized by a monotone gradient, different kinds of generalized convex functions give rise to gradient maps with certain generalized monotonicity properties which are inherited from generalized convexity of the underlying functions. At the same time, it is a useful method in researching variational inequality by means of monotonicity and generalized monotonicity of functions. For corresponding research of monotonicity and generalized monotonicity of functions, one can refer to [7-9]. Similarly, one can research some properties of fuzzy mappings and fuzzy variational inequality by studying their monotonicity. On the other hand, it is worth noting that monotonicity has played a very important role in the study of the existence and solution methods of variational inequality problems. Similarly, one can research fuzzy variational inequality by monotonicity of fuzzy mappings. However, very few investigations have appeared to study monotonicity of fuzzy mappings. Based on the above, we give concepts of monotonicity and generalized monotonicity of fuzzy mappings and discuss relations of generalized monotonicity and generalized convexity.

Vector variational inequality was first introduced and studied by Cottle et al. [10] in finite-dimensional Euclidean spaces. This is a generalization of a scalar variational inequality to the vector case by virtue of multicriteria considering. Since then, the theory with applications for vector variational inequality and vector complementarity problems have been studied and generalized by many authors (see, e.g., [11-21] and the references therein). In particular, in [12], Guang-Ya and Xiao-Qi discussed the existence of a solution to the vector variational inequality and the existence of the solution of the vector complementary problem and obtained some valuable results. It offers some ideal for research of the existence of a solution of other variational inequality problems. However, facing uncertainty is a constant challenge for optimization and decision making. Treating uncertainty with fuzzy 
mathematics results in the study of fuzzy optimization and decision making. Recently, Chang and Zhu [22] introduced the concepts of the variational inequality problem for fuzzy mappings which were later developed by Noor [23-25]. For further research of variational inequalities of fuzzy mappings, one can refer to [26-30]. However, very few investigations have appeared to study fuzzy variational inequalities and the existence of a solution by means of fuzzy numbers. In [5], $\mathrm{Wu}$ and $\mathrm{Xu}$ discussed the relationship between the fuzzy prevariational inequality and the fuzzy vector optimization problem based on nonconvex fuzzy mappings. In [6], Wu and $\mathrm{Xu}$ discussed the relationship between the fuzzy variationallike inequality and the fuzzy vector optimization problem based on generalized convex fuzzy mappings and gave an application example of a fuzzy variational-like inequality: the fuzzy variational-like inequality representation of a fuzzy transportation equilibrium problem. On the other hand, the role of generalized monotonicity of the operator in variational inequality problems corresponds to the role of generalized convexity of the objective function in mathematical programming problems. Similarly, the role of monotonicity of fuzzy mappings should also be very important for research of fuzzy variational inequality problems, particularly research of the existence of a solution of a fuzzy variational inequality. Based on the above, we introduce the fuzzy variational-like inequality, discuss some properties of the fuzzy variationallike inequality, and study the existence of a solution of the fuzzy variational-like inequality by virtue of generalized monotonicity of fuzzy mappings. It is well known that we can solve variational inequality by transforming a variational inequality problem into an optimization problem, for example, [18, 31-33]. Similarly, fuzzy variational inequality also can be transformed into a fuzzy optimization problem. It is difficult to solve a fuzzy optimization problem by means of traditional optimization methods, but a special genetic algorithm can be used to solve fuzzy optimization problems. Therefore, in the sixth section, we discuss the solving of the fuzzy variational-like inequality by virtue of genetic algorithm.

In this paper, we study invex monotonicity and pseudoinvex monotonicity of fuzzy mappings and discuss relations between invex monotonicity (pseudo-invex monotonicity) and invexity (pseudo-invexity) of fuzzy mappings. We discuss the existence of a solution to the fuzzy variationallike inequality. Furthermore, some extended properties of the fuzzy variational-like inequality are researched. Finally, method of solution is discussed based on genetic algorithm.

This paper is organized as follows. Section 2 recalls some definitions and results in reference to fuzzy numbers. Section 3 introduces invex monotonicity and pseudo-invex monotone of fuzzy mapping and discusses some properties. Section 4 defines a class of fuzzy variational inequality: fuzzy variational-like inequality, and discusses the solution existence for the fuzzy variational-like inequality. Section 5 discusses extended qualities of the fuzzy variational-like inequality. Section 6 discusses the method of solving for the fuzzy variational-like inequality and gives a genetic algorithm of a class of the fuzzy variational-like inequality.

\section{Preliminaries}

A fuzzy set of $R^{n}$ is a mapping $\mu: R^{n} \rightarrow[0,1]$. For each such fuzzy set $\mu$, its $\alpha$-cut set is denoted by $[\mu]^{\alpha}=\left\{x \in R^{n}: \mu(x) \geq\right.$ $\alpha\}$ for all $\alpha \in(0,1]$. The support of $\mu$ is denoted by supp $\mu$; that is, $\left\{x \in R^{n} \mid \mu(x)>0\right\}$. The closure of supp $\mu$ is defined as $[\mu]^{0}$.

Definition 1 (see Wu and Xu [6]). A fuzzy number $\mu$ is a fuzzy set with the following properties:

(1) $\mu$ is normal; that is, there exists $x_{0} \in R^{n}$ such that $\mu\left(x_{0}\right)=1$

(2) $\mu$ is convex fuzzy set; that is, $\mu(\lambda x+(1-\lambda) y) \geq$ $\min (\mu(x), \mu(y)), x, y \in R^{n}, \lambda \in[0,1]$;

(3) $[\mu]^{0}$ is compact.

Let $E$ denote the family of fuzzy numbers; that is, $E$ denotes the family of compact and convex fuzzy set on $R^{1}$. Obviously, $[\mu]^{\alpha}$ is a nonempty compact convex subset of $R^{1}$ (denoted by $\left[\mu_{*}(\alpha), \mu^{*}(\alpha)\right]$ ) for all $\mu \in E$ and for all $\alpha \in[0,1]$.

A precise number $a$ is a special case of fuzzy number encoded as

$$
\widetilde{a}(t)= \begin{cases}1, & \text { if } t=a, \\ 0, & \text { if } t \neq a .\end{cases}
$$

However, a precise number will be denoted as usual, in particular, number 0 . The fuzzy numbers $\mu, \nu \in E$ are represented by $\left(\mu_{*}(\alpha), \mu^{*}(\alpha)\right)$ and $\left(\nu_{*}(\alpha), \nu^{*}(\alpha)\right)$, respectively. For each real number $\lambda$, the addition $\mu \widetilde{+} \nu$ and scalar multiplication $\lambda \mu$ are defined as follows:

$$
\begin{gathered}
(\mu \tilde{+} \nu)(x)=\sup _{y+z=x} \min [\mu(y), \nu(z)], \\
(\lambda \mu)(x)=\left\{\begin{array}{ll}
\mu\left(\lambda^{-1} x\right) & \text { if } \lambda \neq 0, \\
0 & \text { if } \lambda=0,
\end{array} \text { for } \mu, \nu \in E, \lambda \in R .\right.
\end{gathered}
$$

It is well known that for all $\mu, \nu \in E$ and $\lambda \in R^{1}$

$$
\begin{gathered}
(\mu \tilde{+} \nu)_{*}(\alpha)=\mu_{*}(\alpha)+v_{*}(\alpha), \\
(\mu \widetilde{+})^{*}(\alpha)=\mu^{*}(\alpha)+\nu^{*}(\alpha), \\
(\lambda \mu)_{*}(\alpha)= \begin{cases}\lambda \mu_{*}(\alpha) & \text { if } \lambda \geq 0, \\
\lambda \mu^{*}(\alpha) & \text { if } \lambda<0,\end{cases} \\
(\lambda \mu)^{*}(\alpha)=\left\{\begin{array}{l}
\lambda \mu^{*}(\alpha) \text { if } \lambda \geq 0, \\
\lambda \mu_{*}(\alpha) \text { if } \lambda<0,
\end{array} \text { for given } \alpha \in[0,1] .\right.
\end{gathered}
$$

For $x=\left(x_{1}, x_{2}, \ldots, x_{n}\right), y=\left(y_{1}, y_{2}, \ldots, y_{n}\right) \in R^{n}, x \leq y$ if and only if $x_{i} \leq y_{i}(i=1,2, \ldots, n)$, and $x<y$ if and only if $x \leq y$ and $x \neq y$.

Definition 2. For $\mu, v \in E, \mu \preceq \nu$, if and only if for every $\alpha \in[0,1], \mu_{*}(\alpha) \leq \nu_{*}(\alpha)$ and $\mu^{*}(\alpha) \leq \nu^{*}(\alpha)$. 


$$
\begin{aligned}
& \text { If } \mu \preceq \nu, \nu \preceq \mu \text {, then } \mu=\nu \text {. } \\
& \mu \prec \nu \text { if and only if } \mu \preceq \nu \text { and } \exists \alpha_{0} \in[0,1] \text {, such that } \\
& \mu_{*}\left(\alpha_{0}\right)<\nu_{*}\left(\alpha_{0}\right) \text { or } \nu_{*}\left(\alpha_{0}\right)<\mu_{*}\left(\alpha_{0}\right) \text {. }
\end{aligned}
$$

For $\mu, v \in E$, if either $\mu \preceq \nu$ or $\nu \preceq \mu$, then $\mu$ and $\nu$ are comparable; otherwise, they are noncomparable.

If $\mu, v \in E$, there exists $\omega \in E$ such that $\mu=\nu \tilde{+} \omega$, then we say the Hukuhara difference of $\mu$ and $\nu$ exists, call $\omega$ the $\mathrm{H}$-difference of $\mu$ and $\nu$, and denote $\mu \simeq \nu=\omega$.

It is obvious that if the H-difference $\mu \simeq v$ exists, then $(\mu \simeq \nu)_{*}(\alpha)=\mu_{*}(\alpha)-\nu_{*}(\alpha),(\mu \simeq \nu)^{*}(\alpha)=\mu^{*}(\alpha)-v^{*}(\alpha)$.

Definition 3. A mapping $F: K\left(\subset R^{n}\right) \rightarrow E$ is said to be a fuzzy mapping. Denote $[F(x)](\alpha)=\left[F(x)_{*}(\alpha), F(x)^{*}(\alpha)\right]$, for all $\alpha \in[0,1]$.

Definition 4 (see Buckley and Feuring [34]). Let $F$ be a fuzzy mapping from the set of real numbers $R$ to the set of all fuzzy numbers, and let $[F(x)](\alpha)=\left[F_{*}(x)(\alpha), F^{*}(x)(\alpha)\right]$. Assume that the partial derivatives of $F_{*}(x)(\alpha), F^{*}(x)(\alpha)$ with respect to $x \in R$ for each $\alpha \in[0,1]$ exist and are denoted by $F_{*}^{\prime}(x, \alpha)$, $F^{* \prime}(x, \alpha)$, respectively. Let $\Gamma(x, \alpha)=\left[F_{*}^{\prime}(x, \alpha), F^{* \prime}(x, \alpha)\right]$ for $x \in R, \alpha \in[0,1]$. If $\Gamma(x, \alpha)$ defines the $\alpha$-cut of a fuzzy number for each $x \in R$, then $F(x)$ is said to be differentiable and is written as $(d F / d x)[\alpha]=\left[F_{*}^{\prime}(x, \alpha), F^{* \prime}(x, \alpha)\right]$, for all $x \in R$, $\alpha \in[0,1]$.

Definition 5 (see Panigrahi et al. [4]). Let $F: K\left(\subset R^{n}\right) \rightarrow$ $E$ be a fuzzy mapping, where $K \subset R^{n}$ is an open set. Let $x=\left(x_{1}, x_{2}, \ldots, x_{n}\right) \in K$. Let $D_{x_{i}},(i=1,2, \ldots, n)$ stand for the "partial differentiation" with respect to the $i$ th variable $x_{i}$. Assume that, for all $\alpha \in[0,1], F_{*}(x)(\alpha), F^{*}(x)(\alpha)$ have continuous partial derivatives so that $D_{x_{i}} F_{*}(x, \alpha), D_{x_{i}} F^{*}(x, \alpha)$ are continuous. Define

$$
\begin{array}{r}
D_{x_{i}} F(x, \alpha)=\left[D_{x_{i}} F_{*}(x, \alpha), D_{x_{i}} F^{*}(x, \alpha)\right], \\
\text { for } i=1,2, \ldots, n, \alpha \in[0,1] .
\end{array}
$$

If each $i=1,2, \ldots, n, D_{x_{i}} F(x, \alpha)$ defines the $\alpha$-cut of a fuzzy number, then $F$ is called differentiable at $x$, and it can be represented as

$$
\widetilde{\nabla} F(x)=\left(D_{x_{1}} F(x, \alpha), D_{x_{2}} F(x, \alpha), \ldots, D_{x_{n}} F(x, \alpha)\right) .
$$

$\widetilde{\nabla} F(x)$ is said to be the gradient of the fuzzy mapping $F$ at $x$.

$U$ is said to be an $n$-dimensional fuzzy vector if and only if the components of $U$ are composed by $n$ fuzzy numbers, denoted by $U=\left(\mu_{1}, \mu_{2}, \ldots, \mu_{n}\right)^{T}$. The set of all $n$-dimensional fuzzy vectors is denoted by $(E)^{n}$.

A $\lambda$ level vector of fuzzy vector $U=\left(\mu_{1}, \mu_{2}, \ldots, \mu_{n}\right)^{T}$ is defined as

$$
\begin{gathered}
{[U]_{\lambda}=\left[\left[\mu_{1}\right]_{\lambda},\left[\mu_{2}\right]_{\lambda}, \ldots,\left[\mu_{n}\right]_{\lambda}\right]^{T},} \\
{[U]_{* \lambda}=\left[\left[\mu_{1}\right]_{* \lambda},\left[\mu_{2}\right]_{* \lambda}, \ldots,\left[\mu_{n}\right]_{* \lambda}\right]^{T},} \\
{[U]_{\lambda}^{*}=\left[\left[\mu_{1}\right]_{\lambda}^{*},\left[\mu_{2}\right]_{\lambda}^{*}, \ldots,\left[\mu_{n}\right]_{\lambda}^{*}\right]^{T} .}
\end{gathered}
$$

The addition and the scalar multiplication of fuzzy vectors $U=\left(\mu_{1}, \mu_{2}, \ldots, \mu_{n}\right)^{T}$ and $V=\left(\nu_{1}, \nu_{2}, \ldots, \nu_{n}\right)^{T}$ are defined as

$$
\begin{array}{r}
U \widetilde{+} V=\left(\mu_{1} \widetilde{+} v_{1}, \mu_{2} \widetilde{+} v_{2}, \ldots, \mu_{n} \widetilde{+} v_{n}\right)^{T}, \\
k U=\left(k \mu_{1} ; k \mu_{2} ; \ldots ; k \mu_{n}\right)^{T}, \quad k \in R, \\
k \mu_{i}=\left\{\left(k \mu_{i *}, k \mu_{i}^{*}, \alpha\right): \alpha \in[0,1]\right\}, \\
\text { for } k>0 .
\end{array}
$$

\section{The Fuzzy Invex Monotone Mapping}

In this section, we put forward some definitions of invex monotonicity of fuzzy mappings and discuss the relationship between invexity and invex monotonicity of fuzzy mappings.

Definition 6. A differentiable comparable fuzzy mapping $F$ : $K \rightarrow E$ is said to be

(a) fuzzy invex with respect to $\eta: K \times K \rightarrow R^{n}$, if and only if

$$
F(x) \geq \eta(x, y)^{T} \nabla F(y) \widetilde{+} F(y), \quad \forall x, y \in K ;
$$

(b) fuzzy incave with respect to $\eta: K \times K \rightarrow R^{n}$, if and only if

$$
F(x) \preceq \eta(x, y)^{T} \nabla F(y) \widetilde{+} F(y), \quad \forall x, y \in K ;
$$

(c) fuzzy strictly invex with respect to $\eta: K \times K \rightarrow R^{n}$, if and only if

$$
F(x)>\eta(x, y)^{T} \nabla F(y) \widetilde{+} F(y), \quad \forall x, y \in K ;
$$

(d) fuzzy strictly incave with respect to $\eta: K \times K \rightarrow R^{n}$, if and only if

$$
F(x) \prec \eta(x, y)^{T} \nabla F(y) \widetilde{+} F(y), \quad \forall x, y \in K ;
$$

(e) fuzzy pseudo-invex with respect to $\eta: K \times K \rightarrow R^{n}$, if and only if

$$
\begin{array}{r}
\eta(x, y)^{T} \nabla F(y) \geq 0 \Longrightarrow F(x) \geq F(y), \\
\forall x, y \in K ;
\end{array}
$$

(f) fuzzy strictly pseudo-invex with respect to $\eta: K \times$ $K \rightarrow R^{n}$, if and only if

$$
\begin{array}{r}
\eta(x, y)^{T} \nabla F(y) \geq 0 \Longrightarrow F(x)>F(y), \\
\forall x, y \in K .
\end{array}
$$

Example 7 (see $\mathrm{Wu}$ and $\mathrm{Xu}[6])$. Let $F: K\left(\subset R^{1}\right) \rightarrow E$ represent the reproduction rate of some germ:

$$
F(x)(t)= \begin{cases}\frac{t}{x^{2}} & t \in\left[0, x^{2}\right], \\ 1-\frac{t-x^{2}}{x^{2}} & t \in\left(x^{2}, 2 x^{2}\right], \\ 0 & t \notin\left[0,2 x^{2}\right] .\end{cases}
$$


So, $[F(x)]^{\alpha}=\left[\alpha x^{2},(2-\alpha) x^{2}\right], \alpha \in[0,1]$. Then there is $\eta(x, y)=x-y$, such that $F(x)$ is a fuzzy invex mapping, where $K=(0, \infty), x, y \in K, x$ represents the predicted quantity, and $t$ represents the actual reproduction quantity.

Example 8. Consider the fuzzy mapping $[F(x)]^{\alpha}=\left[\alpha x^{2},(2-\right.$ $\left.\alpha) x^{2}\right], \alpha \in[0,1]$. Then, there is an $\eta(x, y)=x^{5}-y^{5}$ such that $F(x)$ is a fuzzy pseudoinvex mapping, where $K=(0, \infty)$, $x, y \in K$.

Remark 9. For an invex fuzzy mapping, there must exist $\eta$ : $K \times K \rightarrow R^{n}$, such that

$$
F(x) \geq \eta(x, y)^{T} \nabla F(y) \widetilde{+} F(y), \quad \forall x, y \in K,
$$

holds.

Proof. Since $F$ is a comparable fuzzy mapping, then for all $x, y \in R^{n}$, there is

$$
F(x) \succeq F(y)
$$

or

$$
F(x) \preceq F(y) .
$$

Without loss of generality, suppose that

$$
F(x) \succeq F(y) .
$$

Thus, for all $\alpha \in[0,1]$,

$$
\begin{aligned}
& F_{*}(x)(\alpha) \geq F_{*}(y)(\alpha), \\
& F^{*}(x)(\alpha) \geq F^{*}(y)(\alpha) .
\end{aligned}
$$
holds.

If $\nabla F(y)=0$, for any $\eta(x, y): K \times K \rightarrow R^{n}$, the result

If $\nabla F(y) \neq 0$, for given $\alpha \in[0,1]$.

(1) When $F^{*}(x)(\alpha)-F^{*}(y)(\alpha) \geq F_{*}(x)(\alpha)-F_{*}(y)(\alpha)$.

(i) If $\nabla F_{*}(y)(\alpha)>0$, then take

$$
\begin{aligned}
\eta(x, y)= & \frac{F_{*}(x)(\alpha)-F_{*}(y)(\alpha)}{\left[\nabla F^{*}(y)(\alpha)\right]^{T} \nabla F^{*}(y)(\alpha)} \\
& \times \nabla F^{*}(y)(\alpha) .
\end{aligned}
$$

Thus,

$$
\begin{aligned}
\eta(x, y)^{T} \nabla F^{*}(y)(\alpha) \\
=\frac{F_{*}(x)(\alpha)-F_{*}(y)(\alpha)}{\left[\nabla F^{*}(y)(\alpha)\right]^{T} \nabla F^{*}(y)(\alpha)} \\
\quad \times\left[\nabla F^{*}(y)(\alpha)\right]^{T} \nabla F^{*}(y)(\alpha) \\
=F_{*}(x)(\alpha)-F_{*}(y)(\alpha) \\
\leq F^{*}(x)(\alpha)-F^{*}(y)(\alpha) ;
\end{aligned}
$$

that is,

$$
F^{*}(x)(\alpha) \geq \eta(x, y)^{T} \nabla F^{*}(y)(\alpha)+F^{*}(y)(\alpha) .
$$

From $\nabla F^{*}(y)(\alpha) \geq \nabla F_{*}(y)(\alpha)>0$, there is

$$
\left[\nabla F^{*}(y)(\alpha)\right]^{T}\left(\nabla F^{*}(y)(\alpha)-\nabla F_{*}(y)(\alpha)\right) \geq 0 ;
$$

so,

$$
\begin{aligned}
& {\left[\nabla F^{*}(y)(\alpha)\right]^{T} \nabla F^{*}(y)(\alpha)} \\
& \quad \geq\left[\nabla F^{*}(y)(\alpha)\right]^{T} \nabla F_{*}(y)(\alpha) .
\end{aligned}
$$

Hence,

$$
\begin{aligned}
\eta(x, y)^{T} \nabla F_{*}(y)(\alpha) \\
=\frac{F_{*}(x)(\alpha)-F_{*}(y)(\alpha)}{\left[\nabla F^{*}(y)(\alpha)\right]^{T} \nabla F^{*}(y)(\alpha)} \\
\quad \times\left[\nabla F^{*}(y)(\alpha)\right]^{T} \nabla F_{*}(y)(\alpha) \\
\leq \frac{F_{*}(x)(\alpha)-F_{*}(y)(\alpha)}{\left[\nabla F^{*}(y)(\alpha)\right]^{T} \nabla F^{*}(y)(\alpha)} \\
\quad \times\left[\nabla F^{*}(y)(\alpha)\right]^{T} \nabla F^{*}(y)(\alpha) \\
=F_{*}(x)(\alpha)-F_{*}(y)(\alpha) .
\end{aligned}
$$

That is,

$$
F_{*}(x)(\alpha) \geq \eta(x, y)^{T} \nabla F_{*}(y)(\alpha)+F_{*}(y)(\alpha) .
$$

From (22) and (26), it follows that

$$
F(x) \geq \eta(x, y)^{T} \nabla F(y) \widetilde{+} F(y), \quad \forall x, y \in K .
$$

(ii) If $\nabla F_{*}(y)(\alpha)<0$, then take

$$
\begin{aligned}
\eta(x, y)= & \frac{F_{*}(x)(\alpha)-F_{*}(y)(\alpha)}{\left[\nabla F_{*}(y)(\alpha)\right]^{T} \nabla F_{*}(y)(\alpha)} \\
& \times \nabla F_{*}(y)(\alpha) .
\end{aligned}
$$

Thus,

$$
\begin{aligned}
\eta(x, y)^{T} \nabla F_{*}(y)(\alpha) \\
=\frac{F_{*}(x)(\alpha)-F_{*}(y)(\alpha)}{\left[\nabla F_{*}(y)(\alpha)\right]^{T} \nabla F_{*}(y)(\alpha)} \\
\quad \times\left[\nabla F_{*}(y)(\alpha)\right]^{T} \nabla F_{*}(y)(\alpha) \\
=F_{*}(x)(\alpha)-F_{*}(y)(\alpha) .
\end{aligned}
$$

From $\nabla F_{*}(y)(\alpha)<0$ and $\nabla F^{*}(y)(\alpha) \geq \nabla F_{*}(y)(\alpha)$, there is

$$
\left[\nabla F_{*}(y)(\alpha)\right]^{T}\left(\nabla F^{*}(y)(\alpha)-\nabla F_{*}(y)(\alpha)\right) \leq 0 ;
$$

so,

$$
\begin{aligned}
& {\left[\nabla F_{*}(y)(\alpha)\right]^{T} \nabla F^{*}(y)(\alpha)} \\
& \quad \leq\left[\nabla F_{*}(y)(\alpha)\right]^{T} \nabla F_{*}(y)(\alpha) .
\end{aligned}
$$


Hence,

$$
\begin{aligned}
& \eta(x, y)^{T} \nabla F^{*}(y)(\alpha) \\
&=\frac{F_{*}(x)(\alpha)-F_{*}(y)(\alpha)}{\left[\nabla F_{*}(y)(\alpha)\right]^{T} \nabla F_{*}(y)(\alpha)} \\
& \times\left[\nabla F_{*}(y)(\alpha)\right]^{T} \nabla F^{*}(y)(\alpha) \\
& \leq \frac{F_{*}(x)(\alpha)-F_{*}(y)(\alpha)}{\left[\nabla F_{*}(y)(\alpha)\right]^{T} \nabla F_{*}(y)(\alpha)} \\
& \quad \times\left[\nabla F_{*}(y)(\alpha)\right]^{T} \nabla F_{*}(y)(\alpha) \\
&=F_{*}(x)(\alpha)-F_{*}(y)(\alpha) \\
& \leq F^{*}(x)(\alpha)-F^{*}(y)(\alpha) .
\end{aligned}
$$

That is,

$$
F^{*}(x)(\alpha) \geq \eta(x, y)^{T} \nabla F^{*}(y)(\alpha)+F^{*}(y)(\alpha) .
$$

From (29) and (33), it follows that

$$
F(x) \geq \eta(x, y)^{T} \nabla F(y) \tilde{+} F(y), \quad \forall x, y \in K .
$$

(iii) If $\nabla F_{*}(y)(\alpha)$ is indefinite, there is a vector $C \in R^{n}$, such that

$$
\begin{gathered}
\nabla F_{*}(y)(\alpha)+C \leq 0, \\
C^{T} \nabla F_{*}(y)(\alpha) \leq 0
\end{gathered}
$$

holds.

Take

$$
\begin{aligned}
\eta(x, y)= & \frac{F_{*}(x)(\alpha)-F_{*}(y)(\alpha)}{\left[\nabla F_{*}(y)(\alpha)\right]^{T} \nabla F_{*}(y)(\alpha)} \\
& \times\left(\nabla F_{*}(y)(\alpha)+C\right) .
\end{aligned}
$$

Thus,

$$
\begin{aligned}
& \eta(x, y)^{T} \nabla F_{*}(y)(\alpha) \\
&=\frac{F_{*}(x)(\alpha)-F_{*}(y)(\alpha)}{\left[\nabla F_{*}(y)(\alpha)\right]^{T} \nabla F_{*}(y)(\alpha)} \\
& \quad \times\left[\nabla F_{*}(y)(\alpha)+C\right]^{T} \nabla F_{*}(y)(\alpha) \\
&=F_{*}(x)(\alpha)-F_{*}(y)(\alpha) \\
& \quad+\frac{F_{*}(x)(\alpha)-F_{*}(y)(\alpha)}{\left[\nabla F_{*}(y)(\alpha)\right]^{T} \nabla F_{*}(y)(\alpha)} \\
& \quad \times C^{T} \nabla F_{*}(y)(\alpha) \\
& \leq F_{*}(x)(\alpha)-F_{*}(y)(\alpha) .
\end{aligned}
$$

That is,

$$
F_{*}(x)(\alpha) \geq \eta(x, y)^{T} \nabla F_{*}(y)(\alpha)+F_{*}(y)(\alpha) .
$$

From $\nabla F_{*}(y)(\alpha)+C \leq 0$ and $\nabla F^{*}(y)(\alpha) \geq \nabla F_{*}(y)(\alpha)$, there is

$$
\left[\nabla F_{*}(y)(\alpha)+C\right]^{T}\left(\nabla F^{*}(y)(\alpha)-\nabla F_{*}(y)(\alpha)\right) \leq 0 ;
$$

so,

$$
\begin{aligned}
& {\left[\nabla F_{*}(y)(\alpha)+C\right]^{T} \nabla F^{*}(y)(\alpha)} \\
& \quad \leq\left[\nabla F_{*}(y)(\alpha)+C\right]^{T} \nabla F_{*}(y)(\alpha) .
\end{aligned}
$$

Hence,

$$
\begin{aligned}
& \eta(x, y)^{T} \nabla F^{*}(y)(\alpha) \\
&=\frac{F_{*}(x)(\alpha)-F_{*}(y)(\alpha)}{\left[\nabla F_{*}(y)(\alpha)\right]^{T} \nabla F_{*}(y)(\alpha)} \\
& \times\left[\nabla F_{*}(y)(\alpha)+C\right]^{T} \nabla F^{*}(y)(\alpha) \\
& \leq \frac{F_{*}(x)(\alpha)-F_{*}(y)(\alpha)}{\left[\nabla F_{*}(y)(\alpha)\right]^{T} \nabla F_{*}(y)(\alpha)} \\
& \quad \times\left[\nabla F_{*}(y)(\alpha)+C\right]^{T} \nabla F_{*}(y)(\alpha) \\
&= F_{*}(x)(\alpha)-F_{*}(y)(\alpha) \\
&+\frac{F_{*}(x)(\alpha)-F_{*}(y)(\alpha)}{\left[\nabla F_{*}(y)(\alpha)\right]^{T} \nabla F_{*}(y)(\alpha)} \\
& \times C^{T} \nabla F_{*}(y)(\alpha) \\
& \leq F_{*}(x)(\alpha)-F_{*}(y)(\alpha) \\
& \leq F^{*}(x)(\alpha)-F^{*}(y)(\alpha) .
\end{aligned}
$$

That is,

$$
F^{*}(x)(\alpha) \geq \eta(x, y)^{T} \nabla F^{*}(y)(\alpha)+F^{*}(y)(\alpha) .
$$

From (38) and (42), it follows that

$$
F(x) \succeq \eta(x, y)^{T} \nabla F(y) \widetilde{+} F(y), \quad \forall x, y \in K .
$$

(2) When $F^{*}(x)(\alpha)-F^{*}(y)(\alpha) \leq F_{*}(x)(\alpha)-F_{*}(y)(\alpha)$. (i) If $\nabla F_{*}(y)(\alpha)>0$, then take

$$
\begin{aligned}
\eta(x, y)= & \frac{F^{*}(x)(\alpha)-F^{*}(y)(\alpha)}{\left[\nabla F^{*}(y)(\alpha)\right]^{T} \nabla F^{*}(y)(\alpha)} \\
& \times \nabla F^{*}(y)(\alpha) .
\end{aligned}
$$

Thus,

$$
\begin{aligned}
& \eta(x, y)^{T} \nabla F^{*}(y)(\alpha) \\
& =F^{*}(x)(\alpha)-F^{*}(y)(\alpha) .
\end{aligned}
$$


On the other hand,

$$
\begin{aligned}
& \eta(x, y)^{T} \nabla F_{*}(y)(\alpha) \\
&=\frac{F^{*}(x)(\alpha)-F^{*}(y)(\alpha)}{\left[\nabla F^{*}(y)(\alpha)\right]^{T} \nabla F^{*}(y)(\alpha)} \\
& \quad \times\left[\nabla F^{*}(y)(\alpha)\right]^{T} F_{*}(y)(\alpha) \\
& \leq \frac{F^{*}(x)(\alpha)-F^{*}(y)(\alpha)}{\left[\nabla F^{*}(y)(\alpha)\right]^{T} \nabla F^{*}(y)(\alpha)} \\
& \quad \times\left[\nabla F^{*}(y)(\alpha)\right]^{T} F^{*}(y)(\alpha) \\
&=F^{*}(x)(\alpha)-F^{*}(y)(\alpha) \\
& \leq F_{*}(x)(\alpha)-F_{*}(y)(\alpha) .
\end{aligned}
$$

That is,

$$
\eta(x, y)^{T} \nabla F_{*}(y)(\alpha) \leq F_{*}(x)(\alpha)-F_{*}(y)(\alpha) .
$$

From (45) and (47), it follows that

$$
F(x) \geq \eta(x, y)^{T} \nabla F(y) \widetilde{+} F(y), \quad \forall x, y \in K .
$$

(ii) If $\nabla F_{*}(y)(\alpha)<0$, then take

$$
\begin{aligned}
\eta(x, y)= & \frac{F^{*}(x)(\alpha)-F^{*}(y)(\alpha)}{\left[\nabla F_{*}(y)(\alpha)\right]^{T} \nabla F_{*}(y)(\alpha)} \\
& \times \nabla F_{*}(y)(\alpha) .
\end{aligned}
$$

Thus,

$$
\begin{aligned}
\eta(x, y)^{T} \nabla F_{*}(y)(\alpha) \\
=\frac{F^{*}(x)(\alpha)-F^{*}(y)(\alpha)}{\left[\nabla F_{*}(y)(\alpha)\right]^{T} \nabla F_{*}(y)(\alpha)} \\
\quad \times\left[\nabla F_{*}(y)(\alpha)\right]^{T} \nabla F_{*}(y)(\alpha) \\
=F^{*}(x)(\alpha)-F^{*}(y)(\alpha) \\
\leq F_{*}(x)(\alpha)-F_{*}(y)(\alpha) .
\end{aligned}
$$

That is,

$$
\begin{aligned}
\eta(x, y)^{T} \nabla F_{*}(y)(\alpha) \\
\quad \leq F_{*}(x)(\alpha)-F_{*}(y)(\alpha) .
\end{aligned}
$$

On the other hand,

$$
\begin{aligned}
& \eta(x, y)^{T} \nabla F^{*}(y)(\alpha) \\
&=\frac{F^{*}(x)(\alpha)-F^{*}(y)(\alpha)}{\left[\nabla F_{*}(y)(\alpha)\right]^{T} \nabla F_{*}(y)(\alpha)} \\
& \times\left[\nabla F_{*}(y)(\alpha)\right]^{T} \nabla F^{*}(y)(\alpha) \\
& \leq \frac{F^{*}(x)(\alpha)-F^{*}(y)(\alpha)}{\left[\nabla F_{*}(y)(\alpha)\right]^{T} \nabla F_{*}(y)(\alpha)} \\
& \quad \times\left[\nabla F_{*}(y)(\alpha)\right]^{T} F_{*}(y)(\alpha) \\
&= F^{*}(x)(\alpha)-F^{*}(y)(\alpha) ;
\end{aligned}
$$

that is,

$$
\begin{aligned}
& \eta(x, y)^{T} \nabla F^{*}(y)(\alpha) \\
& \quad \leq F^{*}(x)(\alpha)-F^{*}(y)(\alpha) .
\end{aligned}
$$

From (51) and (53), it follows that

$$
F(x) \succeq \eta(x, y)^{T} \nabla F(y) \widetilde{+} F(y), \quad \forall x, y \in K .
$$

(iii) If $\nabla F_{*}(y)(\alpha)$ is indefinite, there is a vector $C \in R^{n}$, such that

$$
\begin{gathered}
\nabla F_{*}(y)(\alpha)+C \leq 0, \\
C^{T} \nabla F_{*}(y)(\alpha) \leq 0
\end{gathered}
$$

holds.

Take

$$
\begin{aligned}
\eta(x, y)= & \frac{F^{*}(x)(\alpha)-F^{*}(y)(\alpha)}{\left[\nabla F_{*}(y)(\alpha)\right]^{T} \nabla F_{*}(y)(\alpha)} \\
& \times\left(\nabla F_{*}(y)(\alpha)+C\right) .
\end{aligned}
$$

Thus,

$$
\begin{aligned}
& \eta(x, y)^{T} \nabla F_{*}(y)(\alpha) \\
&=\frac{F^{*}(x)(\alpha)-F^{*}(y)(\alpha)}{\left[\nabla F_{*}(y)(\alpha)\right]^{T} \nabla F_{*}(y)(\alpha)} \\
& \quad \times\left[\nabla F_{*}(y)(\alpha)+C\right]^{T} \nabla F_{*}(y)(\alpha) \\
&=F^{*}(x)(\alpha)-F^{*}(y)(\alpha) \\
& \quad+\frac{F^{*}(x)(\alpha)-F^{*}(y)(\alpha)}{\left[\nabla F_{*}(y)(\alpha)\right]^{T} \nabla F_{*}(y)(\alpha)} \\
& \quad \times C^{T} \nabla F_{*}(y)(\alpha) \\
& \leq F^{*}(x)(\alpha)-F^{*}(y)(\alpha) \\
& \leq F_{*}(x)(\alpha)-F_{*}(y)(\alpha) .
\end{aligned}
$$


That is,

$$
\begin{aligned}
& \eta(x, y)^{T} \nabla F_{*}(y)(\alpha) \\
& \quad \leq F_{*}(x)(\alpha)-F_{*}(y)(\alpha) .
\end{aligned}
$$

On the other hand,

$$
\begin{aligned}
& \eta(x, y)^{T} \nabla F^{*}(y)(\alpha) \\
&=\frac{F^{*}(x)(\alpha)-F^{*}(y)(\alpha)}{\left[\nabla F_{*}(y)(\alpha)\right]^{T} \nabla F_{*}(y)(\alpha)} \\
& \times\left[\nabla F_{*}(y)(\alpha)+C\right]^{T} \nabla F^{*}(y)(\alpha) \\
& \leq \frac{F^{*}(x)(\alpha)-F^{*}(y)(\alpha)}{\left[\nabla F_{*}(y)(\alpha)\right]^{T} \nabla F_{*}(y)(\alpha)} \\
& \times\left[\nabla F_{*}(y)(\alpha)+C\right]^{T} F_{*}(y)(\alpha) \\
&< F^{*}(x)(\alpha)-F^{*}(y)(\alpha) ;
\end{aligned}
$$

that is,

$$
\begin{aligned}
& \eta(x, y)^{T} \nabla F^{*}(y)(\alpha) \\
& \quad \leq F^{*}(x)(\alpha)-F^{*}(y)(\alpha) .
\end{aligned}
$$

From (58) and (60), it follows that

$$
\begin{array}{r}
F(x) \geq \eta(x, y)^{T} \nabla F(y) \widetilde{+} F(y), \\
\forall x, y \in K .
\end{array}
$$

This completes the proof.

Definition 10. A comparable fuzzy mapping $F: K \rightarrow(E)^{n}$ is said to be

(a) fuzzy invex monotone on $K$, if $\exists \eta: K \times K \rightarrow R^{n}$ such that for any $x, y \in K$,

$$
\eta(y, x)^{T} F(y) \succeq \eta(y, x)^{T} F(x) ;
$$

(b) fuzzy pseudo-invex monotone on $K$, if $\exists \eta: K \times K \rightarrow$ $R^{n}$ such that for any $x, y \in K$,

$$
\eta(y, x)^{T} F(y) \geq 0 \Longrightarrow \eta(y, x)^{T} F(x) \geq 0 ;
$$

(c) fuzzy strictly invex monotone on $K$, if $\exists \eta: K \times K \rightarrow$ $R^{n}$ such that for any $x, y \in K, x \neq y$,

$$
\eta(y, x)^{T} F(y)>\eta(y, x)^{T} F(x)
$$

(d) fuzzy strictly pseudo-invex monotone if and only if $\exists \eta: K \times K \rightarrow R^{n}$ such that for any $x, y \in K, x \neq y$,

$$
\eta(y, x)^{T} F(y) \geq 0 \Longrightarrow \eta(y, x)^{T} F(x)>0 .
$$

Definition 11. The function $\eta: K \times K \rightarrow R^{n}$ is said to be a skew function if

$$
\eta(x, y)=-\eta(y, x), \quad \forall x, y \in K \text {. }
$$

Definition 12. Let $y \in K, K$ is said to be invex at $y$ with respect to $\eta: K \times K \rightarrow R^{n}$ if, for each $x \in K, \lambda \in[0,1]$,

$$
y+\lambda \eta(x, y) \in K
$$

$K$ is said to be an invex set with respect to $\eta$ if $K$ is invex at each $y \in K$.

Theorem 13. If a differentiable fuzzy mapping $F: K \rightarrow E$ is invex on $K$ with respect to $\eta: K \times K \rightarrow R^{n}$ and $\eta$ is a skew function. Then, $\nabla F: K \rightarrow(E)^{n}$ is fuzzy invex monotone with respect to the same $\eta$.

Proof. Let $F$ be invex on $K$, then there exists $\eta(x, y) \in R^{n}$, such that

$$
F(x) \geq \eta(x, y)^{T} \nabla F(y) \widetilde{\mp} F(y),
$$$$
\forall x, y \in K \text {. }
$$

That is, there are

$$
\begin{aligned}
& F_{*}(x)(\alpha) \geq \eta(x, y)^{T} \nabla F_{*}(y)(\alpha)+F_{*}(y)(\alpha), \\
& F^{*}(x)(\alpha) \geq \eta(x, y)^{T} \nabla F^{*}(y)(\alpha)+F^{*}(y)(\alpha),
\end{aligned}
$$

for all $\alpha \in[0,1]$.

By changing $x$ for $y$,

$$
F(y) \succeq \eta(y, x)^{T} \nabla F(x) \tilde{+} F(x) .
$$

That is, there are

$$
\begin{aligned}
& F_{*}(y)(\alpha) \geq \eta(y, x)^{T} \nabla F_{*}(x)(\alpha)+F_{*}(x)(\alpha), \\
& F^{*}(y)(\alpha) \geq \eta(y, x)^{T} \nabla F^{*}(x)(\alpha)+F^{*}(x)(\alpha),
\end{aligned}
$$

for all $\alpha \in[0,1]$.

From (69) and (71), it follows that

$$
\begin{aligned}
& \eta(x, y)^{T} \nabla F_{*}(y)(\alpha)+\eta(y, x)^{T} \nabla F_{*}(x)(\alpha) \leq 0, \\
& \eta(x, y)^{T} \nabla F^{*}(y)(\alpha)+\eta(y, x)^{T} \nabla F^{*}(x)(\alpha) \leq 0 .
\end{aligned}
$$

As $\eta(x, y)+\eta(y, x)=0$, then

$$
\begin{aligned}
& \eta(y, x)^{T} \nabla F_{*}(y)(\alpha) \geq \eta(y, x)^{T} \nabla F_{*}(x)(\alpha), \\
& \eta(y, x)^{T} \nabla F^{*}(y)(\alpha) \geq \eta(y, x)^{T} \nabla F^{*}(x)(\alpha) .
\end{aligned}
$$

Therefore, from (73), there is

$$
\eta(y, x)^{T} \nabla F(y) \succeq \eta(y, x)^{T} \nabla F(x) .
$$


Corollary 14. If a differentiable fuzzy mapping $F: K \rightarrow E$ is invex on $K$ with respect to $\eta: K \times K \rightarrow R^{n}$ and $\eta$ is a skew function. Then, $\nabla F: K \rightarrow(E)^{n}$ is fuzzy pseudo-invex monotone with respect to the same $\eta$.

Proof. From Theorem 13, it follows that

$$
\eta(y, x)^{T} \nabla F(y) \succeq \eta(y, x)^{T} \nabla F(x), \quad \forall x, y \in K .
$$

Thus,

$$
\begin{aligned}
& \eta(y, x)^{T} \nabla F_{*}(y)(\alpha) \geq \eta(y, x)^{T} \nabla F_{*}(x)(\alpha), \\
& \eta(y, x)^{T} \nabla F^{*}(y)(\alpha) \geq \eta(y, x)^{T} \nabla F^{*}(x)(\alpha),
\end{aligned}
$$

for all $\alpha \in[0,1]$.

If $\eta(y, x)^{T} \nabla F(x) \geq 0$,

$$
\begin{aligned}
& \eta(y, x)^{T} \nabla F^{*}(x)(\alpha) \geq 0, \\
& \eta(y, x)^{T} \nabla F_{*}(x)(\alpha) \geq 0,
\end{aligned}
$$

for all $\alpha \in[0,1]$.

Thus, from (76) and (77),

$$
\begin{aligned}
& \eta(y, x)^{T} \nabla F_{*}(y)(\alpha) \geq 0, \\
& \eta(y, x)^{T} \nabla F^{*}(y)(\alpha) \geq 0
\end{aligned}
$$

holds. Therefore, $\eta(y, x)^{T} \nabla F(y) \geq 0$.

Theorem 15. If a differentiable fuzzy mapping $F: K \rightarrow E$ is strictly invex on $K$ with respect to $\eta: K \times K \rightarrow R^{n}$ and $\eta$ is a skew function. Then, $\nabla F: K \rightarrow(E)^{n}$ is fuzzy strictly invex monotone on $K$ with respect to the same $\eta$.

Proof. Assume that $F$ is strictly invex on $K$, then there exists $\eta(x, y) \in R^{n}$, such that, for any $x, y \in K, x \neq y$,

$$
F(x)>\eta(x, y) \nabla F(y) \tilde{+} F(y) .
$$

Thus, there exists some $\alpha_{0} \in[0,1]$, such that

$$
F_{*}(x)\left(\alpha_{0}\right)>\eta(x, y)^{T} \nabla F_{*}(y)\left(\alpha_{0}\right)+F_{*}(y)\left(\alpha_{0}\right),
$$

or

$$
F^{*}(x)\left(\alpha_{0}\right)>\eta(x, y)^{T} \nabla F^{*}(y)\left(\alpha_{0}\right)+F^{*}(y)\left(\alpha_{0}\right) .
$$

For $\alpha_{0} \in[0,1]$, without loss of generality, suppose that

$$
F_{*}(x)\left(\alpha_{0}\right)>\eta(x, y)^{T} \nabla F_{*}(y)\left(\alpha_{0}\right)+F_{*}(y)\left(\alpha_{0}\right) .
$$

By changing $x$ for $y$,

$$
F_{*}(y)\left(\alpha_{0}\right)>\eta(y, x)^{T} \nabla F_{*}(x)\left(\alpha_{0}\right)+F_{*}(x)\left(\alpha_{0}\right) .
$$

By (82), (83) and since $\eta$ is a skew function, we have

$$
\eta(y, x)^{T} \nabla F_{*}(y)\left(\alpha_{0}\right)>\eta(y, x)^{T} \nabla F_{*}(x)\left(\alpha_{0}\right) .
$$

On the other hand, for other $\alpha \in[0,1]$,

$$
\eta(y, x)^{T} \nabla F(y) \geq \eta(y, x)^{T} \nabla F(x) .
$$

Therefore,

$$
\eta(y, x)^{T} \nabla F(y)>\eta(y, x)^{T} \nabla F(x) .
$$

Theorem 16. If a differentiable fuzzy mapping $F: K \rightarrow E$ is strictly pseudo-invex on $K$ with respect to $\eta: K \times K \rightarrow R^{n}$ and $\eta$ is a skew function. Then, $\nabla F: K \rightarrow(E)^{n}$ is fuzzy strictly pseudo-invex monotone on $K$ with respect to the same $\eta$.

Proof. Let $F$ be a fuzzy strictly pseudo-invex, then there exists $\eta(x, y) \in R^{n}$, such that for any $x, y \in K, x \neq y$,

$$
\eta(x, y)^{T} \nabla F(y) \geq 0 \Longrightarrow F(x)>F(y) .
$$

We need to show that there exists $\eta(x, y) \in R^{n}$, such that

$$
\begin{aligned}
& \eta(x, y)^{T} \nabla F(y) \geq 0 \\
& \Longrightarrow \eta(x, y)^{T} \nabla F(x)>0,
\end{aligned}
$$

for all $x, y \in K, x \neq y$.

By contradiction, suppose that $\eta(x, y)^{T} \nabla F(x) \preceq 0$, then there exists some $\alpha_{0} \in[0,1]$, such that

$$
\eta(x, y)^{T} \nabla F_{*}(x)\left(\alpha_{0}\right) \leq 0,
$$

or

$$
\eta(x, y)^{T} \nabla F^{*}(x)\left(\alpha_{0}\right) \leq 0 .
$$

Without loss of generality, assume that

$$
\eta(x, y)^{T} \nabla F_{*}(x)\left(\alpha_{0}\right) \leq 0 .
$$

As $\eta(x, y)+\eta(y, x)=0$, then

$$
\eta(y, x)^{T} \nabla F_{*}(x)\left(\alpha_{0}\right) \geq 0 .
$$

Since $F$ is strictly pseudo-invex on $K$, then for $\alpha_{0} \in[0,1]$, there is

$$
\begin{aligned}
& \eta(y, x)^{T} \nabla F_{*}(x)\left(\alpha_{0}\right) \geq 0 \\
& \Longrightarrow F_{*}(x)\left(\alpha_{0}\right) \leq F_{*}(y)\left(\alpha_{0}\right),
\end{aligned}
$$

which is a contradiction.

Theorem 17. Let $F: K \rightarrow(E)^{n}$ be a fuzzy strictly pseudo monotone mapping on $K$ with respect to $\eta: K \times K \rightarrow R^{n}$; then, $F$ is a fuzzy pseudo monotone with respect to $\eta$ on $K$.

Proof. As $F$ is a strictly pseudo monotone with respect to $\eta$ on $K$, then for any $\alpha \in[0,1]$,

$$
\begin{aligned}
\eta(y, x)^{T} \nabla F_{*}(x)(\alpha) & \geq 0 \\
\Longrightarrow & F_{*}(x)(\alpha) \leq F_{*}(y)(\alpha), \\
\eta(y, x)^{T} \nabla F^{*}(x)(\alpha) & \geq 0 \\
\Longrightarrow & F_{*}(x)(\alpha) \leq F^{*}(y)(\alpha) .
\end{aligned}
$$


$F_{*}(x)(\alpha), F^{*}(x)(\alpha)$ is strictly pseudo monotone with respect to $\eta$ on $K$. Thus, $F_{*}(x)(\alpha), F^{*}(x)(\alpha)$ also is pseudo monotone with respect to $\eta$ on $K$; that is, $F$ is pseudo monotone with respect to $\eta$ on $K$.

Theorem 18. Let $F: K \rightarrow E$ be a differentiable mapping, and suppose that

(i) $\eta$ satisfies the following conditions:

(a) $\eta(y, y+t \eta(x, y))=-t \eta(x, y)$,

(b) $\eta(x, y+t \eta(x, y))=(1-t) \eta(x, y)$;

(ii) $K$ is an invex set with respect to $\eta$;

(iii) for each $x \neq y$, some $\alpha_{0} \in[0,1]$,

(a) $F_{*}(y)\left(\alpha_{0}\right)>F_{*}(x)\left(\alpha_{0}\right)$ implies $\eta(x, y)^{T} \nabla F_{*}(y+$ $\left.\tilde{\lambda}_{\eta}(x, y)\right)\left(\alpha_{0}\right)<0$,

or

(b) $F^{*}(y)\left(\alpha_{0}\right)>F^{*}(x)\left(\alpha_{0}\right)$ implies $\eta(x, y)^{T} \nabla F^{*}(y+$ $\tilde{\lambda} \eta(x, y))\left(\alpha_{0}\right)<0$

(iv) $\nabla F: K \rightarrow(E)^{n}$ is fuzzy pseudo-invex monotone with respect to $\eta$ on $K$.

Then, $F$ is a fuzzy pseudo-invex mapping $\eta$ on $K$.

Proof. Set $x, y \in K, x \neq y$, and $\eta(x, y)^{T} \nabla F(y) \geq 0$ holds. Thus, we need to show that $F(x) \geq F(y)$; that is, $F_{*}(x)(\alpha) \geq$ $F_{*}(y)(\alpha)$ and $F^{*}(x)(\alpha) \geq F^{*}(y)(\alpha)$, for all $\alpha \in[0,1]$.

Assume the contrary, that is, $F(x) F(y)$. Thus, there exists some $\alpha_{0} \in[0,1]$, such that

$$
F_{*}(x)\left(\alpha_{0}\right)<F_{*}(y)\left(\alpha_{0}\right)
$$

or

$$
F^{*}(x)\left(\alpha_{0}\right)<F^{*}(y)\left(\alpha_{0}\right) .
$$

Without loss of generality, suppose that

$$
F^{*}(x)\left(\alpha_{0}\right)<F^{*}(y)\left(\alpha_{0}\right) .
$$

By hypothesis (iii),

$$
\eta(x, y)^{T} \nabla F^{*}(y+\widetilde{\lambda} \eta(x, y))\left(\alpha_{0}\right)<0,
$$

for some $\tilde{\lambda} \in(0,1)$.

It follows from (98) and (i) that

$$
\begin{aligned}
\eta(y+ & \left.\widetilde{\lambda}_{\eta}(x, y), y\right)^{T} \\
& \times \nabla F^{*}(y+\widetilde{\lambda} \eta(x, y))\left(\alpha_{0}\right)<0,
\end{aligned}
$$

for some $\tilde{\lambda} \in(0,1)$. thus

Since $\nabla F$ is a pseudo-invex monotone with respect to $\eta$,

$$
\eta(y+\tilde{\lambda} \eta(x, y), y)^{T} \nabla F^{*}(y)\left(\alpha_{0}\right)<0 .
$$

From $\eta(y+\tilde{\lambda} \eta(x, y), y)=\tilde{\lambda} \eta(x, y)$ and $\tilde{\lambda} \in(0,1),(100)$ becomes

$$
\eta(x, y)^{T} \nabla F^{*}(y)\left(\alpha_{0}\right)<0 .
$$

This contradicts $\eta(x, y)^{T} \nabla F^{*}(y)(\alpha) \geq 0$.

Theorem 19. Let $F: K \rightarrow E$ be a differentiable mapping, and suppose that

(i) $\eta$ satisfies the following conditions:

(a) $\eta(y, y+t \eta(x, y))=-t \eta(x, y)$,

(b) $\eta(x, y+t \eta(x, y))=(1-t) \eta(x, y)$;

(a) $\eta(y, y+t \eta(x, y))=-t \eta(x, y)$,

(b) $\eta(x, y+t \eta(x, y))=(1-t) \eta(x, y)$;

(ii) $K$ is an invex set with respect to $\eta$;

(iii) for each $x \neq y$, some $\alpha_{0} \in[0,1]$,

(a) $F_{*}(y)\left(\alpha_{0}\right)>F_{*}(x)\left(\alpha_{0}\right)$ implies $\eta(x, y)^{T} \nabla F_{*}(y+$ $\tilde{\lambda} \eta(x, y))\left(\alpha_{0}\right)<0$,

or

(b) $F^{*}(y)\left(\alpha_{0}\right)>F^{*}(x)\left(\alpha_{0}\right)$ implies $\eta(x, y)^{T} \nabla F^{*}(y+$ $\tilde{\lambda} \eta(x, y))\left(\alpha_{0}\right)<0$;

(iv) $\nabla F: K \rightarrow(E)^{n}$ is fuzzy strictly pseudo-invex monotone with respect to $\eta$ on $k$.

Then, $F$ is a fuzzy strictly pseudoinvex mapping $\eta$ on $k$.

Proof. Let $x, y \in K, x \neq y$, such that $\eta(x, y)^{T} \nabla F(y) \geq 0$. Thus, we need to show that $F(x)>F(y)$.

By contradiction, suppose that $F(x) \tilde{r} F(y)$; then, there exists some $\alpha_{0} \in[0,1]$, such that

$$
F_{*}(x)\left(\alpha_{0}\right) \leq F_{*}(y)\left(\alpha_{0}\right)
$$

or

$$
F^{*}(x)\left(\alpha_{0}\right) \leq F^{*}(y)\left(\alpha_{0}\right) .
$$

Without loss of generality, suppose that

$$
F^{*}(x)\left(\alpha_{0}\right) \leq F^{*}(y)\left(\alpha_{0}\right) .
$$

By hypothesis (iii),

$$
\eta(x, y)^{T} \nabla F^{*}\left(y+\widetilde{\lambda}_{\eta}(x, y)\right)\left(\alpha_{0}\right) \leq 0,
$$

for some $\tilde{\lambda} \in(0,1)$.

It follows from (i) and above inequity (105) that

$$
\begin{aligned}
\eta(y, y & +\widetilde{\lambda} \eta(x, y))^{T} \\
& \times \nabla F^{*}(y+\widetilde{\lambda} \eta(x, y))\left(\alpha_{0}\right) \geq 0 .
\end{aligned}
$$


Since $\nabla F$ is a strictly pseudo-invex monotone with respect to $\eta$, thus

$$
\eta(y, y+\widetilde{\lambda} \eta(x, y))^{T} \nabla F^{*}(y)\left(\alpha_{0}\right)>0
$$

From $\eta(y, y+\widetilde{\lambda} \eta(x, y))=-\widetilde{\lambda} \eta(x, y)$ and $\tilde{\lambda} \in(0,1)$, it follows that

$$
\eta(x, y)^{T} \nabla F^{*}(y)\left(\alpha_{0}\right)<0 .
$$

This contradicts $\eta(x, y)^{T} \nabla F^{*}(y)(\alpha) \geq 0$.

\section{The Existence of a Solution to the Fuzzy Variational-Like Inequality}

Let $K \subset R^{n}, \eta\left(x, x^{*}\right): K \times K \rightarrow R^{n}, F: K \rightarrow(E)^{n}$, the fuzzy variational-like inequality problem be: find $x^{*} \in K, F\left(x^{*}\right) \in$ $(E)^{n}$ (denoted by $\left.F\left(x^{*}\right)=\left(F_{1}\left(x^{*}\right), F_{2}\left(x^{*}\right), \ldots, F_{n}\left(x^{*}\right)\right)^{n}\right)$, such that

$$
\eta\left(x, x^{*}\right)^{T} F\left(x^{*}\right) \prec 0, \quad \forall x \in K .
$$

Definition 20. Let $K$ be an invex set with respect to $\eta\left(x, x^{*}\right)$ : $K \times K \rightarrow R^{n}$. A fuzzy mapping $F: K \rightarrow(E)^{n}$ is called $\eta$-hemicontinuous, if for $x, y \in K$, for all $\alpha \in[0,1]$, the mappings $t \rightarrow F^{*}(y+\operatorname{t\eta }(x, y))(\alpha)$ and $t \rightarrow F_{*}(y+$ $\operatorname{t\eta }(x, y))(\alpha)$ are continuous at $0^{+}$, with $t \in[0,1]$.

Lemma 21. Let $K$ be a nonempty convex set in $R^{n}$, and suppose that

(i) $F: K \rightarrow(E)^{n}$ is a fuzzy pseudo-invex monotone with respect to $\eta$ and $\eta$-hemicontinuous on $K$;

(ii) $\eta: K \times K \rightarrow R^{n}$ satisfies

(a) $\eta(y, y+\lambda \eta(x, y))=-\lambda \eta(x, y)$, for all $x, y \in K$, $\lambda \in[0,1]$,

(b) $\eta(x, y+\lambda \eta(x, y))=(1-\lambda) \eta(x, y)$, for all $x, y \in$ $K, \lambda \in[0,1]$;

(iii) for any fixed $y \in K, x \rightarrow \eta(x, y)$ is linear; that is, for $x^{(i)} \in K, i=1,2, \ldots, n, t_{i} \in[0,1], \sum_{i=1}^{n} t_{i}=1$, with $\eta\left(\sum_{i=1}^{n} t_{i} x^{(i)}, y\right)=\sum_{i=1}^{n} t_{i} \eta\left(x^{(i)}, y\right)$

Then, for $y \in K, \eta(x, y)^{T} F(y) \prec 0$, for all $x \in K$ if and only if $\eta(x, y)^{T} F(x) \prec 0$, for all $x \in K$.

Proof. $\Rightarrow$ By contradiction, suppose that there exists a $\bar{x} \epsilon$ $K$, such that $\eta(\bar{x}, y)^{T} F(\bar{x}) \prec 0$. Thus, there exists some $\alpha_{0} \in$ $[0,1]$,

$$
\eta(\bar{x}, y)^{T} F_{*}(\bar{x})\left(\alpha_{0}\right)<0
$$

or

$$
\eta(\bar{x}, y)^{T} F^{*}(\bar{x})\left(\alpha_{0}\right)<0 .
$$

Without loss of generality, assume that

$$
\eta(\bar{x}, y)^{T} F^{*}(\bar{x})\left(\alpha_{0}\right)<0 .
$$

Since $F: K \rightarrow(E)^{n}$ is a fuzzy pseudo-invex monotone with respect to $\eta$, thus

$$
\begin{array}{rl}
\eta(\bar{x}, y)^{T} & F(y) \geq 0 \\
& \Longrightarrow \eta(\bar{x}, y)^{T} F(\bar{x}) \geq 0 .
\end{array}
$$

That is, there are

$$
\begin{aligned}
& \eta(\bar{x}, y)^{T} F^{*}(y)(\alpha) \geq 0 \\
& \Longrightarrow(\bar{x}, y)^{T} F^{*}(\bar{x})(\alpha) \geq 0, \\
& \eta(\bar{x}, y)^{T} F_{*}(y)(\alpha) \geq 0 \\
& \Longrightarrow \eta(\bar{x}, y)^{T} F_{*}(\bar{x})(\alpha) \geq 0,
\end{aligned}
$$

for all $\alpha \in[0,1]$.

Therefore,

$$
\begin{aligned}
\eta(\bar{x}, y)^{T} F^{*}(\bar{x})(\alpha)<0 \\
\Longrightarrow \eta(\bar{x}, y)^{T} F^{*}(y)(\alpha)<0, \\
\eta(\bar{x}, y)^{T} F_{*}(\bar{x})(\alpha)<0 \\
\Longrightarrow \eta(\bar{x}, y)^{T} F_{*}(y)(\alpha)<0 .
\end{aligned}
$$

In particular, for $\alpha_{0} \in[0,1]$,

$$
\begin{aligned}
\eta(\bar{x}, y)^{T} F^{*}(\bar{x})\left(\alpha_{0}\right)<0 \\
\Longrightarrow \eta(\bar{x}, y)^{T} F^{*}(y)\left(\alpha_{0}\right)<0 .
\end{aligned}
$$

This contradicts $\eta(x, y)^{T} F(y) \bar{\prec} 0$.

$\Leftarrow$ By contradiction, suppose that there exists a $\bar{x} \in K$, such that $\eta(\bar{x}, y)^{T} F(\bar{y}) \prec 0$. Thus, there exists some $\alpha_{0} \in$ $[0,1]$,

$$
\eta(\bar{x}, y)^{T} F_{*}(y)\left(\alpha_{0}\right)<0
$$

or

$$
\eta(\bar{x}, y)^{T} F^{*}(y)\left(\alpha_{0}\right)<0 .
$$

Without loss of generality, assume that

$$
\eta(\bar{x}, y)^{T} F^{*}(y)\left(\alpha_{0}\right)<0 .
$$

Since $K$ is an invex set and by condition (a), we knowthat

$$
y+t(\bar{x}-y) \in K, \quad t \in[0,1], \eta(y, y)=0 .
$$

When $\eta$ satisfies (iii), there is

$$
\begin{aligned}
\eta(y+ & t(\bar{x}-y), y) \\
& =t \eta(\bar{x}, y)+(1-t) \eta(y, y) \\
& =t \eta(\bar{x}, y), \quad t \in[0,1] .
\end{aligned}
$$


Since $F$ is $\eta$-hemicontinuous on $K$, it follows that

$$
\begin{gathered}
t \longrightarrow 0^{+}, \\
F^{*}(y+t(\bar{x}-y))\left(\alpha_{0}\right) \longrightarrow F^{*}(y)\left(\alpha_{0}\right) .
\end{gathered}
$$

Therefore,

$$
\begin{aligned}
& \eta(\bar{x}, y)^{T} F^{*}(y)\left(\alpha_{0}\right)<0 \\
& \Longrightarrow \eta(y+t(\bar{x}-y), y)^{T} \\
& \times F^{*}(y+t(\bar{x}-y))\left(\alpha_{0}\right)<0 .
\end{aligned}
$$

Let $\widehat{x}=y+t(\bar{x}-y)$, then

$$
\eta(\widehat{x}, y)^{T} F^{*}(\widehat{x})\left(\alpha_{0}\right)<0 .
$$

This contradicts $\eta(x, y)^{T} F(x) \prec 0$, for all $x \in K$.

By the hypothesis of the pseudo-invex monotonicity of $F$ and the linearity of $\eta$, the existence theorem can be obtained.

Theorem 22. Let $K$ be a nonempty convex set in $R^{n}$, suppose that

(i) $F: K \rightarrow(E)^{n}$ is fuzzy pseudo-invex monotone with respect to $\eta$ and $\eta$-hemicontinuous on $K$;

(ii) $\eta: K \times K \rightarrow R^{n}$ satisfies

(a) $\eta(y, y+\lambda \eta(x, y))=-\lambda \eta(x, y)$, for all $x, y \in K$, $\lambda \in[0,1]$,

(b) $\eta(x, y+\lambda \eta(x, y))=(1-\lambda) \eta(x, y)$, for all $x, y \in$ $K, \lambda \in[0,1]$;

(iii) for any fixed $y \in K, x \rightarrow \eta(x, y)$ is linear; that is, for $x^{(i)} \in K, i=1,2, \ldots, n, t_{i} \in[0,1], i=1,2, \ldots, n$, for $\sum_{i=1}^{n} t_{i}=1$, with $\eta\left(\sum_{i=1}^{n} t_{i} x^{(i)}, y\right)=\sum_{i=1}^{n} t_{i} \eta\left(x^{(i)}, y\right)$.

Then, there exists $x^{*} \in K$, such that $\eta\left(x, x^{*}\right)^{T} F\left(x^{*}\right) \prec 0$, for all $x \in K$.

Proof. Let

$$
\begin{array}{r}
Y(y)=\left\{x \in K \mid \eta(y, x)^{T} F(x) \prec 0\right\}, \\
\forall y \in K .
\end{array}
$$

That is, for all $\alpha \in[0,1]$, there are

$$
\begin{array}{r}
Y^{*}(y)(\alpha)=\left\{x \in K \mid \eta(y, x)^{T} F^{*}(x)(\alpha) \overline{<} 0\right\}, \\
\forall y \in K, \\
Y_{*}(y)(\alpha)=\left\{x \in K \mid \eta(y, x)^{T} F_{*}(x)(\alpha) \overline{<} 0\right\}, \\
\forall y \in K .
\end{array}
$$

Let $x^{(i)} \in K, i=1,2, \ldots, n, t_{i} \in[0,1], \sum_{i=1}^{n} t_{i}=1$. Suppose that

$$
x=\sum_{i=1}^{n} t_{i} x^{(i)} \bar{\epsilon} \bigcup Y^{*}\left(x^{(i)}\right)(\alpha) .
$$

Then, there exists some $\alpha_{0} \in[0,1]$, such that

$$
\begin{array}{r}
\eta\left(x^{(i)}, x\right)^{T} F^{*}(x)(\alpha)<0, \\
i=1,2, \ldots, n .
\end{array}
$$

Therefore,

$$
\sum_{i=1}^{n} t_{i} \eta\left(x^{(i)}, x\right)^{T} F^{*}(x)(\alpha)<0 .
$$

From (iii), for fixed $x \in K$,

$$
\eta\left(\sum_{i=1}^{n} t_{i} x^{(i)}, x\right)^{T} F^{*}(x)(\alpha)<0 .
$$

That is,

$$
\eta(x, x)^{T} F^{*}(x)(\alpha)<0 .
$$

From $\eta(x, x)=0$, it follows that $0<0$, which is absurd. So $x=\sum_{i=1}^{n} t_{i} x^{(i)} \in \bigcup Y^{*}\left(x^{(i)}\right)(\alpha)$.

Similarly, $x=\sum_{i=1}^{n} t_{i} x^{(i)} \in \bigcup Y_{*}\left(x^{(i)}\right)(\alpha)$ can be proofed. Thus,

$$
\begin{aligned}
& \operatorname{conv}\left(\left\{x^{(1)}, x^{(2)}, \ldots, x^{(n)}\right\}\right) \subset \bigcup Y^{*}\left(x^{(i)}\right)(\alpha), \\
& \operatorname{conv}\left(\left\{x^{(1)}, x^{(2)}, \ldots, x^{(n)}\right\}\right) \subset \bigcup Y_{*}\left(x^{(i)}\right)(\alpha)
\end{aligned}
$$

can be obtained. Therefore, $Y^{*}(y)(\alpha), Y_{*}(y)(\alpha)$ are KKM mappings.

Let

$$
\bar{Y}(y)=\left\{x \in K \mid \eta(y, x)^{T} F(y) \prec 0\right\}, \quad \forall y \in K .
$$

That is, for all $\alpha \in[0,1]$,

$$
\begin{array}{ll}
\bar{Y}^{*}(y)=\left\{x \in K \mid \eta(y, x)^{T} F^{*}(y)(\alpha)<0\right\}, & \forall y \in K, \\
\bar{Y}_{*}(y)=\left\{x \in K \mid \eta(y, x)^{T} F_{*}(y)(\alpha)<0\right\}, & \forall y \in K .
\end{array}
$$

Let $x \in Y(y)$; that is, $\eta(y, x)^{T} F(x) \prec 0$. By Lemma 21, $\eta(y, x)^{T} F(y) \bar{\prec} 0$ holds, that is, $x \in \bar{Y}(y)$. Thus,

$$
\begin{aligned}
& Y^{*}(y)(\alpha) \subset \bar{Y}^{*}(y)(\alpha), \\
& Y_{*}(y)(\alpha) \subset \bar{Y}_{*}(y)(\alpha) .
\end{aligned}
$$

As $Y^{*}(y)(\alpha), Y_{*}(y)(\alpha)$ are KKM mappings, thus $\bar{Y}^{*}(y)(\alpha)$, $\bar{Y}_{*}(y)(\alpha)$ are also KKM mappings. By Lemma 21, we have

$$
\begin{aligned}
& \bigcap_{y \in K} Y^{*}(y)(\alpha)=\bigcap_{y \in K} \bar{Y}^{*}(y)(\alpha), \\
& \bigcap_{y \in K} Y_{*}(y)(\alpha)=\bigcap_{y \in K} \bar{Y}_{*}(y)(\alpha) .
\end{aligned}
$$


As $\bar{Y}_{*}(y)(\alpha), \bar{Y}^{*}(y)(\alpha)$ are closed for every $y \in K$ and $K$ is a bounded set, then $\bar{Y}_{*}(y)(\alpha), \bar{Y}^{*}(y)(\alpha)$ are bounded; hence, $\bar{Y}_{*}(y)(\alpha), \bar{Y}^{*}(y)(\alpha)$ are compact. Therefore,

$$
\begin{aligned}
& \bigcap_{y \in K} Y^{*}(y)(\alpha)=\bigcap_{y \in K} \bar{Y}^{*}(y)(\alpha) \neq \emptyset, \\
& \bigcap_{y \in K} Y_{*}(y)(\alpha)=\bigcap_{y \in K} \bar{Y}_{*}(y)(\alpha) \neq \emptyset .
\end{aligned}
$$

Hence, there exists $x^{*} \in K$, such that

$$
\begin{aligned}
& \eta\left(y, x^{*}\right)^{T} F^{*}\left(x^{*}\right)(\alpha) \overline{<} 0, \quad \forall y \in K, \\
& \eta\left(y, x^{*}\right)^{T} F_{*}\left(x^{*}\right)(\alpha) \overline{<} 0, \quad \forall y \in K .
\end{aligned}
$$

That is,

$$
\eta\left(y, x^{*}\right)^{T} F\left(x^{*}\right) \prec 0, \quad \forall y \in K .
$$

The pseudo-invex monotonicity of $F$ assures us of the existence of a solution to (FVLI), but not the uniqueness of such a solution. To achieve this, we assume the strictly pseudo-invex monotonicity of $F$.

Theorem 23. Let $K$ be a nonempty convex set in $R^{n}$, and suppose that

(i) $F: K \rightarrow(E)^{n}$ is strictly fuzzy pseudo-invex monotone with respect to $\eta$ and $\eta$-hemicontinuous on $K$;

(ii) $\eta: K \times K \rightarrow R^{n}$ is a skew function and satisfies

(a) $\eta(y, y+\lambda \eta(x, y))=-\lambda \eta(x, y)$, for all $x, y \in K$, $\lambda \in[0,1]$,

(b) $\eta(x, y+\lambda \eta(x, y))=(1-\lambda) \eta(x, y)$, for all $x, y \in$ $K, \lambda \in[0,1]$

(iii) for any fixed $y \in K, x \rightarrow \eta(x, y)$ is linear; that is, for $x^{(i)} \in K, i=1,2, \ldots, n, t_{i} \in[0,1], \sum_{i=1}^{n} t_{i}=1$, with $\eta\left(\sum_{i=1}^{n} t_{i} x^{(i)}, y\right)=\sum_{i=1}^{n} t_{i} \eta\left(x^{(i)}, y\right)$

Then, there exists unique $x^{*} \in K$, such that $\eta\left(x, x^{*}\right)^{T} F\left(x^{*}\right) \prec 0$, for all $x \in K$.

Proof. From Theorem 17, $F$ is a fuzzy pseudo-invex monotone. Also from Theorem 22, there exists a solution for problem (FVLI).

Suppose that (FVLI) has two distinct solutions $x^{*}, \bar{x}$. Then,

$$
\begin{gathered}
\eta\left(\bar{x}, x^{*}\right)^{T} F\left(x^{*}\right) \prec 0, \\
\eta\left(x^{*}, \bar{x}\right)^{T} F(\bar{x}) \prec 0 .
\end{gathered}
$$

Since $F$ is a fuzzy strictly pseudo-invex monotone on $K$, then

$$
\begin{aligned}
\eta\left(\bar{x}, x^{*}\right)^{T} F\left(x^{*}\right) & \geq 0 \\
& \Longrightarrow \eta\left(\bar{x}, x^{*}\right)^{T} F(\bar{x})>0 .
\end{aligned}
$$

That is, there are

$$
\begin{aligned}
& \eta\left(\bar{x}, x^{*}\right)^{T} F^{*}\left(x^{*}\right)(\alpha) \geq 0 \\
& \Longrightarrow \eta\left(\bar{x}, x^{*}\right)^{T} F^{*}(\bar{x})(\alpha)>0, \\
& \eta\left(\bar{x}, x^{*}\right)^{T} F_{*}\left(x^{*}\right)(\alpha) \geq 0 \\
& \Longrightarrow \eta\left(\bar{x}, x^{*}\right)^{T} F_{*}(\bar{x})(\alpha)>0,
\end{aligned}
$$

for all $\alpha \in[0,1]$. Thus

Since $\eta$ is a skew function, there is $\eta\left(\bar{x}, x^{*}\right)+\eta\left(x^{*}, \bar{x}\right)=0$.

$$
\begin{aligned}
& \eta\left(\bar{x}, x^{*}\right)^{T} F^{*}\left(x^{*}\right)(\alpha) \geq 0 \\
& \Longrightarrow\left(x^{*}, \bar{x}\right)^{T} F^{*}(\bar{x})(\alpha)<0, \\
& \eta\left(\bar{x}, x^{*}\right)^{T} F_{*}\left(x^{*}\right)(\alpha) \geq 0 \\
& \Longrightarrow \eta\left(x^{*}, \bar{x}\right)^{T} F_{*}(\bar{x})(\alpha)<0 .
\end{aligned}
$$

That is,

$$
\eta\left(x^{*}, \bar{x}\right)^{T} F(\bar{x}) \prec 0,
$$

which contradicts $\eta\left(x^{*}, \bar{x}\right)^{T} F(\bar{x}) \prec 0$.

\section{Qualities of the Fuzzy Variational-Like Inequality}

A well-known fact in mathematical programming is that the variational inequality problem has a close relationship with the optimization problem. Similarly, the fuzzy variational inequality problem also has a close relationship with the fuzzy optimization problem.

Consider the unconstrained fuzzy vector optimization problem:

$$
\min _{x \in K} F(x)
$$

where $K$ is a subset of $\mathrm{n}$ dimension Euclidean space $R^{n}, F$ : $K \rightarrow E$ is a fuzzy mapping.

A point $x \in K$ is called a feasible point. If $x^{*} \in K$ and no $x \in K, F(x) \prec F\left(x^{*}\right)$, then $x^{*}$ is called an optimal solution, a global optimal solution, or simply a solution to the problem (FP). If $x^{*} \in K$ and there exists an $\delta$-neighborhood $N_{\delta}\left(x^{*}\right)$ around $x^{*}$, such that for no $x\left(\neq x^{*}\right) \in K \cap N_{\delta}\left(x^{*}\right), F(x) \prec$ $F\left(x^{*}\right)$, then $x^{*}$ is called a local optimal solution. Similarly, if $x^{*} \in K$ and there exists an $\delta$-neighborhood $N_{\delta}\left(x^{*}\right)$ around $x^{*}$, such that for no $x \in K \cap N_{\delta}\left(x^{*}\right), F(x) \preceq F\left(x^{*}\right)$, then $x^{*}$ is called a strict local optimal solution.

The following lemmas and theorems discuss the properties of fuzzy variational inequality.

Lemma 24 (see $\mathrm{Wu}$ and $\mathrm{Xu}[6]$ ). Let $F: K \rightarrow E$ be a fuzzy differentiable pseudoinvex mapping. If $\left(x^{*}, \nabla F\left(x^{*}\right)\right)$ is a solution of $(F V L I)$, then $x^{*}$ is a local optimal solution of $(F P)$. 
Lemma 25 (see $\mathrm{Wu}$ and $\mathrm{Xu}[6])$. Let $F: K \rightarrow E$ be a fuzzy differentiable strictly pseudoinvex mapping. If $\left(x^{*}, \nabla F\left(x^{*}\right)\right)$ is a solution of (FVLI), then $x^{*}$ is a strictly local optimal solution of $(F P)$.

Theorem 26. Let $F: K \rightarrow E$ be a differentiable fuzzy mapping, and suppose that

(i) $\eta$ satisfies the following conditions:

(a) $\eta(y, y+t \eta(x, y))=-t \eta(x, y)$

(b) $\eta(x, y+t \eta(x, y))=(1-t) \eta(x, y)$;

(ii) $K$ is an invex set with respect to $\eta$;

(iii) for each $x \neq y$, some $\alpha_{0} \in[0,1]$,

(a) $F_{*}(y)\left(\alpha_{0}\right)>F_{*}(x)\left(\alpha_{0}\right)$ implies $\eta(x, y)^{T} \nabla F_{*}(y+$ $\tilde{\lambda} \eta(x, y))\left(\alpha_{0}\right)<0$,

or

(b) $F^{*}(y)\left(\alpha_{0}\right)>F^{*}(x)\left(\alpha_{0}\right)$ implies $\eta(x, y)^{T} \nabla F^{*}(y+$ $\tilde{\lambda} \eta(x, y))\left(\alpha_{0}\right)<0$

(iv) $\nabla F: K \rightarrow(E)^{n}$ is fuzzy pseudo-invex monotone with respect to $\eta$ on $k$.

If $\left(x^{*}, \nabla F\left(x^{*}\right)\right)$ is a solution of $(F V L I)$, then $x^{*}$ is a local optimal solution of $(F P)$.

Proof. From Theorem 18, we know that $F$ is a fuzzy pseudo invex. By Lemma 24, we can show it.

Theorem 27. Let $F: K \rightarrow E$ be a differentiable fuzzy mapping, and suppose that

(i) $\eta$ satisfies the following conditions:

(a) $\eta(y, y+t \eta(x, y))=-t \eta(x, y)$,

(b) $\eta(x, y+t \eta(x, y))=(1-t) \eta(x, y)$;

(ii) $K$ is an invex set with respect to $\eta$;

(iii) for each $x \neq y$, some $\alpha_{0} \in[0,1]$,

(a) $F_{*}(y)\left(\alpha_{0}\right)>F_{*}(x)\left(\alpha_{0}\right)$ implies $\eta(x, y)^{T} \nabla F_{*}(y+$ $\tilde{\lambda} \eta(x, y))\left(\alpha_{0}\right)<0$,

or

(b) $F^{*}(y)\left(\alpha_{0}\right)>F^{*}(x)\left(\alpha_{0}\right)$ implies $\eta(x, y)^{T} \nabla F^{*}(y+$ $\tilde{\lambda} \eta(x, y))\left(\alpha_{0}\right)<0$

(iv) $\nabla F: K \rightarrow(E)^{n}$ is fuzzy strictly pseudo-invex monotone with respect to $\eta$ on $k$.

If $\left(x^{*}, \nabla F\left(x^{*}\right)\right)$ is a solution of $(F V L I)$, then $x^{*}$ is a strictly local optimal solution of $(F P)$.

Proof. From Theorem 19, we know that $F$ is a fuzzy strictly pseudo-invex. By Lemma 25, we can show it.
Theorem 28 gives the equivalent relationship of two fuzzy variational-like inequalities.

Theorem 28. Let $K$ be an invex set with respect to $\eta: K \times$ $K \rightarrow R^{n}$. Suppose that

(i) $F: K \rightarrow(E)^{n}$ is fuzzy pseudo-invex monotone with respect to $\eta$ and $\eta$-hemicontinuous on $K$;

(ii) $\eta$ satisfies the following conditions:

(a) $\eta(y, y+\lambda \eta(x, y))=-\lambda \eta(x, y)$,

(b) $\eta(x, y+\lambda \eta(x, y))=(1-\lambda) \eta(x, y)$.

Then, $x^{*} \in K$ satisfies $\eta\left(x, x^{*}\right)^{T} F\left(x^{*}\right) \bar{\prec} 0$, for all $x \in K$ if and only if it satisfies $\eta\left(x, x^{*}\right)^{T} F(x) \prec 0$, for all $x \in K$.

Proof. $\Rightarrow$ By contradiction, suppose that there exists an $\bar{x} \epsilon$ $K$, such that $\eta\left(\bar{x}, x^{*}\right)^{T} F(\bar{x}) \prec 0$. Thus, there exists some $\alpha_{0} \in$ $[0,1]$,

$$
\eta\left(\bar{x}, x^{*}\right)^{T} F^{*}(\bar{x})\left(\alpha_{0}\right)<0
$$

or

$$
\eta\left(\bar{x}, x^{*}\right)^{T} F_{*}(\bar{x})\left(\alpha_{0}\right)<0 .
$$

Without loss of generality, assume that

$$
\eta\left(\bar{x}, x^{*}\right)^{T} F^{*}(\bar{x})\left(\alpha_{0}\right)<0 .
$$

Since $F: K \rightarrow(E)^{n}$ is fuzzy pseudo-invex monotone with respect to $\eta$, thus

$$
\begin{aligned}
\eta\left(\bar{x}, x^{*}\right)^{T} F\left(x^{*}\right) & \geq 0 \\
& \Longrightarrow \eta\left(\bar{x}, x^{*}\right)^{T} F(\bar{x}) \geq 0 .
\end{aligned}
$$

That is, for any $\alpha \in[0,1]$,

$$
\begin{gathered}
\eta\left(\bar{x}, x^{*}\right)^{T} F^{*}\left(x^{*}\right)(\alpha) \geq 0 \\
\Longrightarrow \eta\left(\bar{x}, x^{*}\right)^{T} F^{*}(\bar{x})(\alpha) \geq 0, \\
\eta\left(\bar{x}, x^{*}\right)^{T} F_{*}\left(x^{*}\right)(\alpha) \geq 0 \\
\Longrightarrow \eta\left(\bar{x}, x^{*}\right)^{T} F_{*}(\bar{x})(\alpha) \geq 0 .
\end{gathered}
$$

Therefore,

$$
\begin{gathered}
\eta\left(\bar{x}, x^{*}\right)^{T} F^{*}(\bar{x})(\alpha)<0 \\
\Longrightarrow \eta\left(\bar{x}, x^{*}\right)^{T} F^{*}\left(x^{*}\right)(\alpha)<0, \\
\eta\left(\bar{x}, x^{*}\right)^{T} F_{*}(\bar{x})(\alpha)<0 \\
\Longrightarrow \eta\left(\bar{x}, x^{*}\right)^{T} F_{*}\left(x^{*}\right)(\alpha)<0 .
\end{gathered}
$$

In particularly, for $\alpha_{0} \in[0,1]$, there is

$$
\begin{aligned}
\eta\left(\bar{x}, x^{*}\right)^{T} F^{*}(\bar{x})\left(\alpha_{0}\right)<0 \\
\quad \Longrightarrow \eta\left(\bar{x}, x^{*}\right)^{T} F^{*}\left(x^{*}\right)\left(\alpha_{0}\right)<0 .
\end{aligned}
$$

This contradicts $\eta\left(\bar{x}, x^{*}\right)^{T} F\left(x^{*}\right) \bar{\prec} 0$. 
$\Leftarrow$ By contradiction, suppose that there exists an $\bar{x} \in K$, such that $\eta\left(\bar{x}, x^{*}\right)^{T} F\left(x^{*}\right) \prec 0$. Thus, there exists some $\alpha_{0} \in$ $[0,1]$,

$$
\eta\left(\bar{x}, x^{*}\right)^{T} F^{*}\left(x^{*}\right)\left(\alpha_{0}\right)<0,
$$

or

$$
\eta\left(\bar{x}, x^{*}\right)^{T} F_{*}\left(x^{*}\right)\left(\alpha_{0}\right)<0 .
$$

Without loss of generality, assume that

$$
\eta\left(\bar{x}, x^{*}\right)^{T} F^{*}\left(x^{*}\right)\left(\alpha_{0}\right)<0 .
$$

Since $K$ is invex set with respect to $\eta$ and assumption (ii), it follows that

$$
\begin{aligned}
x^{*}+\lambda \eta\left(\bar{x}, x^{*}\right) & \in K, \\
\eta\left(x^{*}+\lambda \eta\left(\bar{x}, x^{*}\right), x^{*}\right)= & \lambda \eta\left(\bar{x}, x^{*}\right), \\
& \lambda \in[0,1] .
\end{aligned}
$$

Again, since $F$ is $\eta$-hemicontinuous on $K$, there is

$$
\begin{gathered}
\lambda \longrightarrow 0^{+}, \\
F^{*}\left(x^{*}+\lambda \eta\left(\bar{x}, x^{*}\right)\right)\left(\alpha_{0}\right) \longrightarrow F^{*}\left(x^{*}\right)\left(\alpha_{0}\right) .
\end{gathered}
$$

Therefore,

$$
\begin{aligned}
\eta\left(\bar{x}, x^{*}\right)^{T} F^{*}\left(x^{*}\right)\left(\alpha_{0}\right)<0 \\
\Longrightarrow \\
\Longrightarrow \eta\left(x^{*}+\lambda \eta\left(\bar{x}, x^{*}\right)\right)^{T} \\
\quad \times F^{*}\left(x^{*}+\lambda \eta\left(\bar{x}, x^{*}\right)\right)\left(\alpha_{0}\right)<0 .
\end{aligned}
$$

Set $\widetilde{x}=x^{*}+\lambda \eta\left(\bar{x}, x^{*}\right)$, then

$$
\eta\left(\tilde{x}, x^{*}\right)^{T} F^{*}(\tilde{x})\left(\alpha_{0}\right)<0, \quad \tilde{x} \in K .
$$

This contradicts $\eta\left(x, x^{*}\right)^{T} F(x) \overline{<}$, for all $x \in K$.

\section{Solution to the Fuzzy \\ Variational-Like Inequality}

In order to solve the fuzzy variational-like inequality, it is important find an equivalent fuzzy problem. Next, we discuss the equivalent fuzzy generalized complementarity problem of (FVLI), where $\eta(y, x)=y-x$.

Denote (FVLI): find $x \in K \subset R^{n}, F(x) \in(E)^{n}$, such that

$$
F(x)^{T}(y-x) \prec 0, \quad \forall y \in K,
$$

where $F: K \rightarrow(E)^{n}$.

It is well known that variational inequalities are equivalent to the generalized complementary problem over a convex cone [35]. Similarly, we consider solving the fuzzy variationallike inequality in view of the following fuzzy generalized complementarity problem.
Find $x \in K \subset R^{n}, F(x) \in(E)^{n}$, such that

$$
\begin{gathered}
F(x)^{T} x=0, \\
F(x) \in K^{*}, \\
K^{*}=\left\{z \in(E)^{n} \mid z^{T} y<0, \forall y \in K\right\} .
\end{gathered}
$$

The following theorem shows the equivalence between the fuzzy variational-like inequality and the fuzzy generalized complementarity problem.

Theorem 29. The fuzzy variational-like inequality (FVLI) is equivalent to the fuzzy generalized complementarity problem $(F C P)$, when $K$ is a convex cone.

Proof. At first, we show $(\mathrm{FCP}) \subset(\mathrm{FVLI})$. Suppose that $\left(x^{*}, F\left(x^{*}\right)\right)$ is a solution of (FCP), then

$$
\begin{gathered}
F\left(x^{*}\right)^{T} x^{*}=0, \\
F\left(x^{*}\right)^{T} y \prec 0, \quad \forall y \in K .
\end{gathered}
$$

Combining (158) and (159), we have

$$
F\left(x^{*}\right)^{T}\left(y-x^{*}\right) \bar{\prec}, \quad \forall y \in K .
$$

Therefore, $\left(x^{*}, F\left(x^{*}\right)\right)$ is also a solution of (FVLI).

Next, we show $(\mathrm{FVLI}) \subset(\mathrm{FCP})$. Let $\left(x^{*}, F\left(x^{*}\right)\right)$ be a solution of (FVLI) which the degree of membership $\alpha \in[0,1]$, then

$$
F\left(x^{*}\right)^{T}\left(y-x^{*}\right) \geq \mu_{C_{y}}^{-1}(\alpha) \geq-\widetilde{p}, \quad \forall y \in K,
$$

where $\widetilde{p} \geq 0$ is the tolerance level which a decision maker can tolerate in the accomplishment of the fuzzy variation-like inequality $F(x)^{T}(y-x) \overline{<} 0$. By contradiction, suppose that $F\left(x^{*}\right)^{T} x^{*} \neq 0$, then there exists some $\alpha_{0} \in[0,1], \tilde{q}_{1} \geq 0$, such that

$$
\left[F\left(x^{*}\right)^{T} x^{*}\right]^{*}\left(\alpha_{0}\right)>\tilde{q}_{1}^{*}\left(\alpha_{0}\right)
$$

or

$$
\left[F\left(x^{*}\right)^{T} x^{*}\right]_{*}\left(\alpha_{0}\right)>\widetilde{q}_{1_{*}}\left(\alpha_{0}\right) .
$$

Or there exists some $\alpha_{1} \in[0,1], \widetilde{q}_{2}>0$, such that

$$
\left[F\left(x^{*}\right)^{T} x^{*}\right]^{*}\left(\alpha_{1}\right)<\tilde{q}_{2}^{*}\left(\alpha_{1}\right)
$$

or

$$
\left[F\left(x^{*}\right)^{T} x^{*}\right]_{*}\left(\alpha_{1}\right)>\widetilde{q}_{2_{*}}\left(\alpha_{1}\right) .
$$

Without loss of generality, suppose that

$$
\begin{aligned}
& {\left[F\left(x^{*}\right)^{T} x^{*}\right]^{*}\left(\alpha_{0}\right)>\tilde{q}_{1}^{*}\left(\alpha_{0}\right),} \\
& {\left[F\left(x^{*}\right)^{T} x^{*}\right]^{*}\left(\alpha_{1}\right)<\tilde{q}_{2}^{*}\left(\alpha_{1}\right) .}
\end{aligned}
$$


Since $K$ is a convex cone, then when $y=\lambda x^{*}$ with $\lambda>1$, there is

$$
F\left(x^{*}\right)^{T} x^{*} \geq \frac{-\tilde{p}}{\lambda-1}, \quad \widetilde{p} \geq 0 .
$$

When $y=0$,

$$
F\left(x^{*}\right)^{T} x^{*} \preceq \tilde{p} .
$$

If $\left[F\left(x^{*}\right)^{T} x^{*}\right]^{*}\left(\alpha_{0}\right)>\tilde{q}_{1}^{*}\left(\alpha_{0}\right)$, then

$$
\left[\frac{-\widetilde{p}}{\lambda-1}\right]^{*}\left(\alpha_{0}\right)>\tilde{p}_{1}^{*}\left(\alpha_{0}\right) .
$$

This leads to a contradiction.

$$
\text { If }\left[F\left(x^{*}\right)^{T} x^{*}\right]_{*}\left(\alpha_{1}\right)<\widetilde{q}_{2_{*}}\left(\alpha_{1}\right) \text {, then }
$$

$$
\widetilde{p}^{*}\left(\alpha_{1}\right)<\widetilde{q}_{2_{*}}\left(\alpha_{1}\right)
$$

This also leads to a contradiction. Therefore, $F\left(x^{*}\right)^{T} x^{*}=0$. Furthermore, from (161), it follows that

$$
\begin{aligned}
F\left(x^{*}\right)^{T} & \left(y-x^{*}\right) \geq-\widetilde{p} \\
& \Longrightarrow F\left(x^{*}\right)^{T} y \geq-\widetilde{p}, \quad \forall y \in K .
\end{aligned}
$$

Therefore,

$$
F\left(x^{*}\right)^{T} y \prec 0 .
$$

This shows that $\left(x^{*}, F\left(x^{*}\right)\right)$ is a solution of (FCP).

To solve (FVLI) with $K=\left\{x \in R^{n} \mid D x \geq o, D \in\right.$ $R^{m \times n}$, and $D=\left[d_{i}\right]$, for all $\left.i=1,2, \ldots, m\right\}$ being a convex cone, we consider the problem (FCP) with $d_{i} x \geq 0$, for all $i=1,2, \ldots, m$.

That is,

$$
\begin{gathered}
d_{i} x \geq 0, \quad F(x)^{T} x=0, \\
F(x) \in K^{*}, \quad \forall i=1,2, \ldots, m,
\end{gathered}
$$

where $K^{*}=\left\{z \in(E)^{n} \mid z^{T y} \prec 0\right.$, for all $\left.y \in K\right\}$.

Similarly [26], it can be shown that (173) can be rewritten as follows: find $x \in K, F(x) \in(E)^{n}$ such that

$$
\begin{gathered}
d_{i} x \geq 0, \quad F(x)^{T} x=0, \\
d_{i}^{\prime} F(x) \geq 0, \quad \forall i=1,2, \ldots, m,
\end{gathered}
$$

where $d_{i}^{\prime}$ is a normal to $d_{i}$. Of course, (174) can be also rewritten as follows: find $x \in K, F(x) \in(E)^{n}$ such that

$$
\begin{gathered}
d_{i} x \geq 0, \quad F(x)^{T} x \geq 0, \\
-F(x)^{T} x \geq 0, \\
d_{i}^{\prime} F(x) \geq 0, \quad \forall i=1,2, \ldots, m .
\end{gathered}
$$

In (174), each fuzzy inequality can be represented by a fuzzy set $S_{j}$ with the corresponding membership function $\mu_{s_{j}}(x)$, for $j=1,2, \ldots, 2 m+2$; that is

$$
\begin{aligned}
& \mu_{j}(x)= \begin{cases}1, & \text { if } d_{j} x \geq 0, \\
\mu_{j}\left(d_{j} x\right), & \text { if }-t_{j} \prec d_{j} x \leq 0, \forall j=1,2, \ldots, m, \\
0, & \text { if } d_{j} x \leq-t_{j},\end{cases} \\
& \mu_{s_{m+1}}(x)= \begin{cases}1, & \text { if } F(x)^{T} x \geq 0, \\
\mu_{m+1}\left(F(x)^{T} x\right), & \text { if }-t_{m+1} \prec F(x)^{T} x \preceq 0, \\
0, & \text { if } F(x)^{T} x \preceq-t_{m+1},\end{cases} \\
& \mu_{s_{m+2}}(x)= \begin{cases}1, & \text { if }-F(x)^{T} x \geq 0, \\
\mu_{m+2}\left(-F(x)^{T} x\right), & \text { if }-t_{m+1} \prec-F(x)^{T} x \leq 0, \\
0, & \text { if }-F(x)^{T} x \preceq-t_{m+2},\end{cases} \\
& \mu_{j}(x)= \begin{cases}1, & \text { if } d_{j-m-2}^{\prime} x \geq 0, \\
\mu_{j}\left(d_{j-m-2}^{\prime} x\right), & \text { if }-t_{j} \prec d_{j-m-2}^{\prime} \preceq 0, \\
0, & \forall j=m+3, m+4, \ldots, 2 m+2,\end{cases}
\end{aligned}
$$

where $t_{j} \succeq 0$, for all $j=1,2, \ldots, m$ are the tolerance level of $d_{j} x \geq 0, j=1,2, \ldots, m$. $t_{j} \geq 0$, for all $j=m+1, m+$ $2, \ldots, 2 m+2$ other fuzzy inequalities in which $t_{j}$ are fuzzy numbers.

To find a solution to problem (175), we define a fuzzy decision $\widetilde{D}$ of (175) as the fuzzy set resulting from the intersection of fuzzy set $s_{j}, j=1,2, \ldots, 2 m+2$. By choosing the commonly used "minimum operator" for the fuzzy set intersections, we can define the membership function for $\widetilde{D}$ as

$$
\mu_{j}=\min _{j}\left\{\mu_{s_{j}}(x)\right\}
$$

Therefore, a solution to problem (175) can be taken as the solution with the highest membership in the fuzzy decision set $\widetilde{D}$ and obtained by solving the following problem:

$$
\max _{x} \mu_{j}=\max _{x} \min _{j=1,2, \ldots, 2 m+2}\left\{\mu_{s_{j}}(x)\right\} .
$$

Problem (178) is an unconstrained optimization, but its objective function is not continuous and derivable. It cannot be solved by traditional optimization methods, but it may be solved by genetic algorithms [36, 37]. Next, we discuss a special genetic algorithm with mutation along the weighted gradient direction developed by Wang and Tang [37-39].

For individual $x$, let $\mu_{\min }(x)=\min \left\{\mu_{s_{1}}(x), \mu_{s_{2}}(x)\right.$, $\left.\ldots, \mu_{s_{2 m+2}}(x)\right\}$. If $\mu_{\text {min }}(x) \leq \mu_{s_{j}}(x)<1$, then move along the gradient direction of $\mu_{s_{j}}(x)$, and the value of $\mu_{s_{j}}(x)$ may be improved. The smaller $\mu_{s_{j}}(x)$ is, the greater the improvement in $\mu_{s_{j}}(x)$ that may be achieved. Based on the above idea, construct the direction as follows:

$$
G(x)=\sum_{j=1}^{2 m+2} w_{j} \nabla \mu_{s_{j}}(x),
$$

where $0<\mu_{s_{j}}(x) \leq 1$. 
$G(x)$ is called the weighted gradient direction of $\mu_{\widetilde{D}}$, and $w_{j}$ is the gradient direction weight defined as follows:

$$
w_{j}= \begin{cases}0, & \text { if } \mu_{s_{j}}(x)=1, \\ \frac{1}{\mu_{s_{j}}-\mu_{\min }+\epsilon}, & \text { if } \mu_{\min } \leq \mu_{s_{j}}<1,\end{cases}
$$

where $\epsilon$ is a sufficiently small positive number and $1 / \epsilon$ is the largest weight.

The child $x_{j}^{k+1}$ generated from $x_{i}^{k}$ by mutation along the weighted gradient direction $G(x)$ can be described as follows:

$$
x_{j}^{k+1}=x_{i}^{k}+\beta^{k} G\left(x_{i}^{k}\right)
$$

where $\beta^{k}$ is a random step length of the Erlang distribution generated by a random number generator with declining means. The degree of membership is calculated as follows:

$$
\mu_{s_{j}}\left(x^{(j)}\right)= \begin{cases}\mu_{\min }\left(x^{(j)}\right), & \text { if } \mu_{\min }>\alpha_{0}, \\ \epsilon \mu_{\min }\left(x^{(j)}\right), & \text { else, }\end{cases}
$$

where $\alpha_{0}$ is the acceptable satisfaction degree preferred by the decision maker and $\epsilon \in U(0,1)$.

For individual $j(j=1,2, \ldots$, pop-size $)$, calculate its fitness function $F(j)$ and selected probability $P(j)$ by

$$
\begin{gathered}
F(j)=\mu_{s_{j}}\left(x^{(j)}\right)+\widetilde{\epsilon}, \\
P(j)=\frac{F(j)}{\sum_{j=1}^{\text {pop-size }} F(j)},
\end{gathered}
$$

where $\widetilde{\epsilon}$ is a small positive number. It is used to guarantee a nonzero denominator. The procedure can be written as follows.

Step 1. Transforming (FVLI) into problem (175).

Step 2. Initialize.

(i) Input an acceptable satisfaction degree $\alpha_{0}$ and the largest max-gen and pop-size.

(ii) Input the criteria set: $C_{I}=1,2, \ldots, n, n+1, \ldots, n+$ $2 m+2, n+2 m+3$, where $j=1,2, \ldots, n$ stand for the variables, $j=n+1, \ldots, n+2 m+2$ stand for inequality constraints to (175), and $j=n+2 m+3$ stands for the sum of weighted satisfaction degree, respectively. Give the initial values and the upper and the lower values of criteria $r, r_{i} \in C_{I}$.

(iii) Input the types of membership function that describes the fuzzy constraints.

(iv) Input the weights $w_{j}$.

Step 3. Randomly produce the initial population and calculate their membership degrees by $x_{i}(j)=\xi x_{i}^{\text {up }}$, where $\xi \epsilon$ $U(0,1), i=1,2, \ldots, n ; j=1,2, \ldots$, pop-size and $x_{i}^{\text {up }}$ is the upper bound of the $i$ th element of variable $x$. The membership function $\mu_{S_{j}}\left(x^{(j)}\right)$ is calculated with (182).
Step 4. Set iteration index $k=1$.

Step 5. Calculate the fitness function $F(j)$ and selection probabilities $P(j)$ by means of (183).

Step 6. Produce new individual $x^{(j)}$ with the parent $x^{(i)}$ as $x^{(j) k}=x^{(i)(k-1)}+\beta^{k} G\left(x^{(i)(k-1)}\right) \cdot G(x)$ is defined by (179).

Step 7. For individual $j$, calculate the membership function $\mu_{S_{j}}\left(x^{(j)}\right)$ with (182), and update optimal degree of membership $\mu_{\max }$ and the upper and the lower values of criteria $r$.

Step 8. Set $k+1=k$; if $k \leq$ max-gen, go to Step 5; otherwise, go to Step 9.

Step 9. Output the optimal membership degree $\mu_{\max }$ and the upper and the lower values of criteria preferred by the decision maker, then stop.

\section{Conclusion}

In this paper, we have introduced the concepts of invex monotonicity and pseudoinvex monotonicity of fuzzy mapping and discussed the relationship between invex monotonicity(pseudoinvex monotonicity) and invexity (pseudoinvexity) of fuzzy mapping. We have put forward the fuzzy variational-like inequality and also discussed extended qualities of it. Finally, we discussed the existence of a theorem for a solution and solving method to the fuzzy variationallike inequality. We got some useful results and gave a method of solving the fuzzy variational-like inequality of a genetic algorithm.

\section{Acknowledgments}

This research was supported by the Key Program of NSFC (Grant no. 70831005); the National Science Foundation for Distinguished Young Scholars, China (Grant no. 70425005); The Young and Middle-Aged Leader Scientific Research Foundation of Chengdu University of Information Technology (J201218), China; Bringing in Qualified Personnel Projects of Chengdu University of Information Technology (KYTZ201203), China.

\section{References}

[1] S. S. L. Chang and L. A. Zadeh, "On fuzzy mapping and control," IEEE Transactions on Systems, Man, and Cybernetics, vol. 2, pp. 30-34, 1972.

[2] S. Nanda and K. Kar, "Convex fuzzy mappings," Fuzzy Sets and Systems., vol. 48, no. 1, pp. 129-132, 1992.

[3] H. Yan and J. Xu, "A class of convex fuzzy mappings," Fuzzy Sets and Systems, vol. 129, no. 1, pp. 47-56, 2002.

[4] M. Panigrahi, G. Panda, and S. Nanda, "Convex fuzzy mapping with differentiability and its application in fuzzy optimization," European Journal of Operational Research, vol. 185, no. 1, pp. 4762, 2008.

[5] Z. Wu and J. Xu, "Nonconvex fuzzy mappings and the fuzzy prevariational inequality," Fuzzy Sets and Systems. An International 
Journal in Information Science and Engineering, vol. 159, no. 16, pp. 2090-2103, 2008.

[6] Z. Wu and J. Xu, "Generalized convex fuzzy mappings and fuzzy variational-like inequality," Fuzzy Sets and Systems, vol. 160, no. 11, pp. 1590-1619, 2009.

[7] G. Ruiz-Garzón, R. Osuna-Gómez, and A. Rufián-Lizana, "Generalized invex monotonicity," European Journal of Operational Research, vol. 144, no. 3, pp. 501-512, 2003.

[8] J.-P. Crouzeix and J. A. Ferland, "Criteria for differentiable generalized monotone maps," Mathematical Programming, vol. 75, no. 3, pp. 399-406, 1996.

[9] A. Daniilidis and N. Hadjisavvas, "On the subdifferentials of quasiconvex and pseudoconvex functions and cyclic monotonicity," Journal of Mathematical Analysis and Applications, vol. 237, no. 1, pp. 30-42, 1999.

[10] R. W. Cottle, F. Giannessi, and J.-L. Lions, Eds., Theorems of Alternative, Quadratic Programs and Complementary Problems, Variational Inequalities and Complementary Problems, John Wiley \& Sons, New York, NY, USA, 1980.

[11] G. Ruiz-Garzón, R. Osuna-Gómez, and A. Rufián-Lizana, "Relationships between vector variational-like inequality and optimization problems," European Journal of Operational Research, vol. 157, no. 1, pp. 113-119, 2004.

[12] C. Guang-Ya and Y. Xiao-Qi, "The vector complementary problem and its equivalences with the weak minimal element in ordered spaces," Journal of Mathematical Analysis and Applications, vol. 153, no. 1, pp. 136-158, 1990.

[13] G. Y. Chen, "Existence of solutions for a vector variational inequality: an extension of the Hartmann-Stampacchia theorem," Journal of Optimization Theory and Applications, vol. 74, no. 3, pp. 445-456, 1992.

[14] G.-Y. Chen and B. D. Craven, "Approximate dual and approximate vector variational inequality for multiobjective optimization," Australian Mathematical Society A, vol. 47, no. 3, pp. 418423, 1989.

[15] G. Chen and G. Cheng:, Vector Variational Inequality and Vector Optimization, vol. 285 of Lecture Notes in Economics and Mathematics Systems, Springer, New York, NY, USA, 1986.

[16] F. Giannessi, Variational Inequalities and Vector Equilibrium, Kluwer Academic Publishers, London, UK, 2000.

[17] D. E. Ward and G. M. Lee, "On relations between vector optimization problems and vector variational inequalities," Journal of Optimization Theory and Applications, vol. 113, no. 3, pp. 583-596, 2002.

[18] X. Q. Yang, "On the gap functions of prevariational inequalities," Journal of Optimization Theory and Applications, vol. 116, no. 2, pp. 437-452, 2003.

[19] X. Q. Yang, "Generalized convex functions and vector variational inequalities," Journal of Optimization Theory and Applications, vol. 79, no. 3, pp. 563-580, 1993.

[20] X. Q. Yang, "Vector variational inequality and vector pseudolinear optimization," Journal of Optimization Theory and Applications, vol. 95, no. 3, pp. 729-734, 1997.

[21] N. J. Huang, X. Q. Yang, and W. K. Chan, "Vector complementarity problems with a variable ordering relation," European Journal of Operational Research, vol. 176, no. 1, pp. 15-26, 2007.

[22] S.-S. Chang and Y.-G. Zhu, "On variational inequalities for fuzzy mappings," Fuzzy Sets and Systems, vol. 32, no. 3, pp. 359-367, 1989.

[23] M. A. Noor, "Variational inequalities for fuzzy mappings. (I)," Fuzzy Sets and Systems, vol. 55, no. 3, pp. 309-312, 1993.
[24] M. A. Noor, "Variational inequalities for fuzzy mapping (II)," Fuzzy Sets and Systems, vol. 97, no. 1, pp. 101-107, 1998.

[25] M. A. Noor, "Variational inequalities for fuzzy mappings. (III)," Fuzzy Sets and Systems, vol. 110, no. 1, pp. 101-108, 2000.

[26] C.-F. Hu, "Solving variational inequalities in a fuzzy environment," Journal of Mathematical Analysis and Applications, vol. 249, no. 2, pp. 527-538, 2000.

[27] C.-F. Hu, "Generalized variational inequalities with fuzzy relation," Journal of Computational and Applied Mathematics, vol. 146, no. 1, pp. 47-56, 2002.

[28] J. Y. Park and J. U. Jeong, "Generalized strongly quasivariational inequalities for fuzzy mappings," Fuzzy Sets and Systems, vol. 99, no. 1, pp. 115-120, 1998.

[29] J. Y. Park, S. Y. Lee, and J. U. Jeong, "Completely generalized strongly quasivariational inequalities for fuzzy mappings," Fuzzy Sets and Systems, vol. 110, no. 1, pp. 91-99, 2000.

[30] J. Zhu, C.-K. Zhong, and G.-P. Wang, "Vector-valued variational principle in fuzzy metric space and its applications," Fuzzy Sets and Systems, vol. 119, no. 2, pp. 343-354, 2001.

[31] G. Chen, C. Goh, and X. Yang, "On gap function for vector variational inequalities," European Journal of Operational Research, vol. 82, pp. 55-72, 2000.

[32] G. Y. Chen and S. J. Li, "Existence of solutions for a generalized vector quasivariational inequality," Journal of Optimization Theory and Applications, vol. 90, no. 2, pp. 321-334, 1996.

[33] X. Q. Yang and J. C. Yao, "Gap functions and existence of solutions to set-valued vector variational inequalities," Journal of Optimization Theory and Applications, vol. 115, no. 2, pp. 407417, 2002.

[34] J. J. Buckley and T. Feuring, "Fuzzy differential equations," Fuzzy Sets and Systems, vol. 110, no. 1, pp. 43-54, 2000.

[35] H.-F. Wang and H.-L. Liao, "Variational inequality with fuzzy convex cone," Journal of Global Optimization, vol. 14, no. 4, pp. 395-414, 1999.

[36] J. H. Holland, Adaptation in Natural and Artificial Systems, University of Michigan Press, Ann Arbor, Mich, USA, 1975.

[37] J. Tang and D. Wang, "An interactive approach based on a genetic algorithm for a type of quadratic programming problems with fuzzy objective and resources," Computers \& Operations Research, vol. 24, no. 5, pp. 413-422, 1997.

[38] J. Tang and D. Wang, "Modelling and optimization for a type of fuzzy nonlinear programming problems in manufacturing systems," in Proceedings of the 35th IEEE Conference on Decision and Control, pp. 4401-4405, December 1996.

[39] D. Wang, "An inexact approach for linear programming with a GA for a type of quardratic programming problem with fuzzy objective and resources," Fuzzy Sets and Systems, vol. 24, pp. 261-281, 1995. 


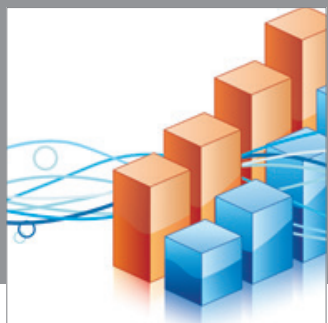

Advances in

Operations Research

mansans

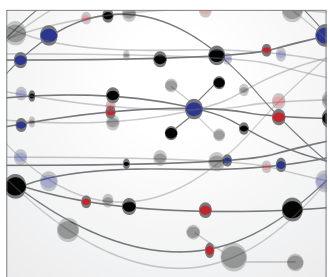

The Scientific World Journal
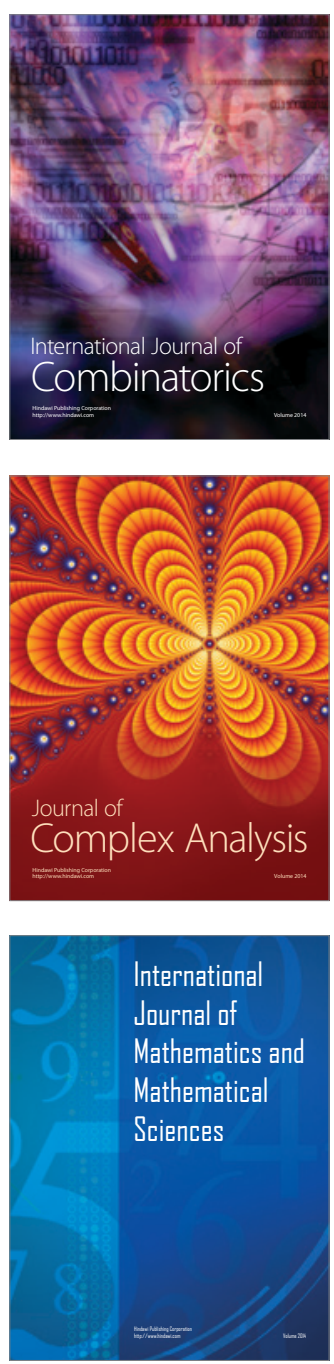
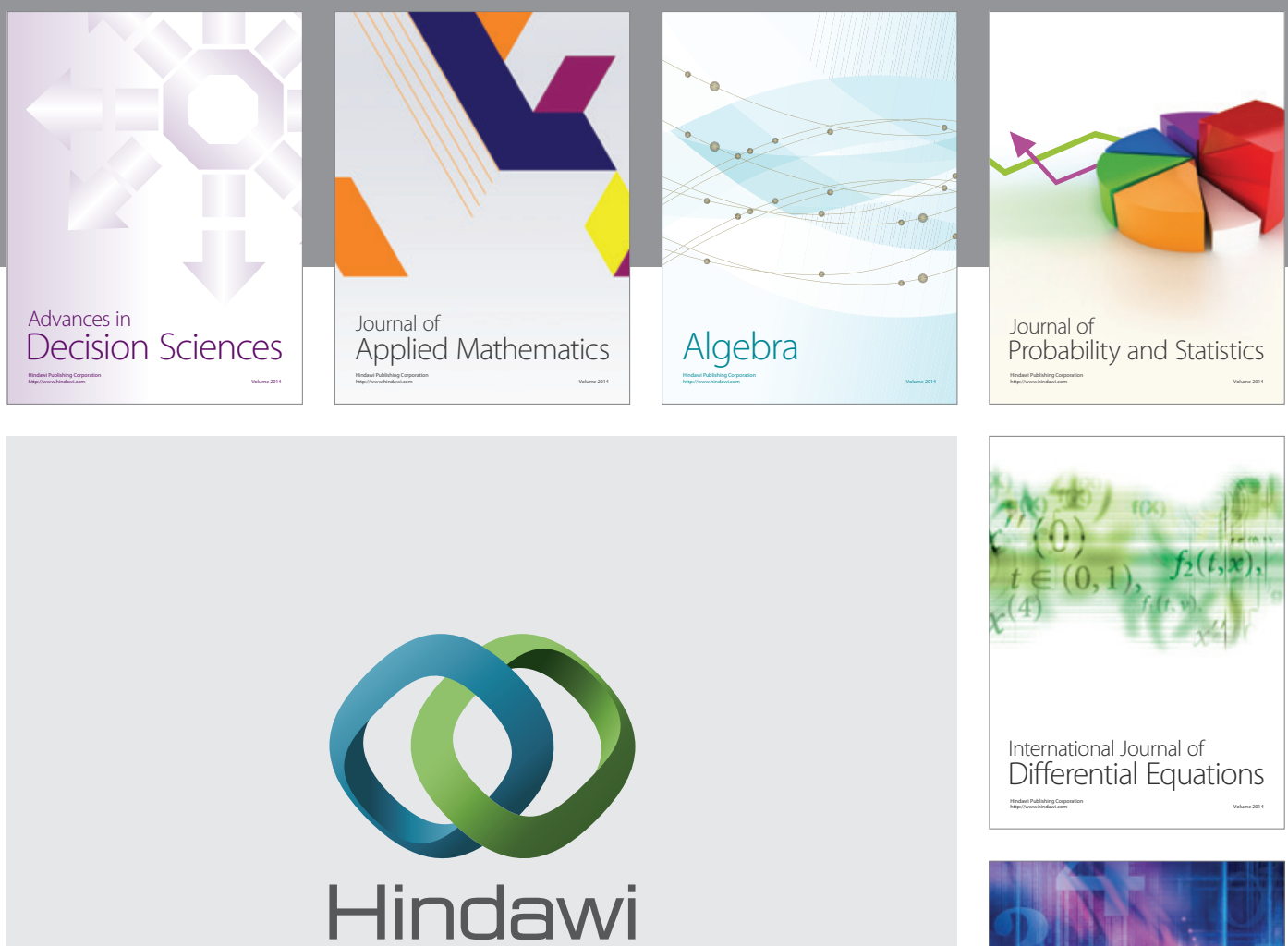

Submit your manuscripts at http://www.hindawi.com
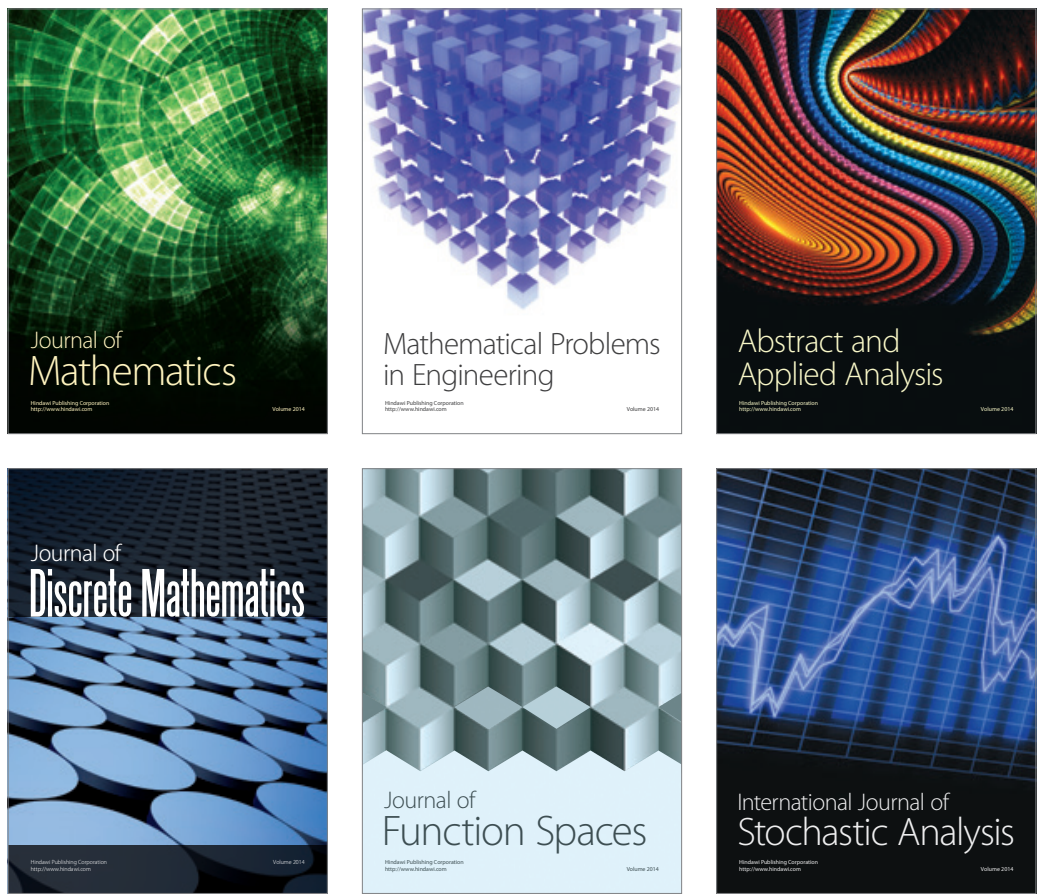

Journal of

Function Spaces

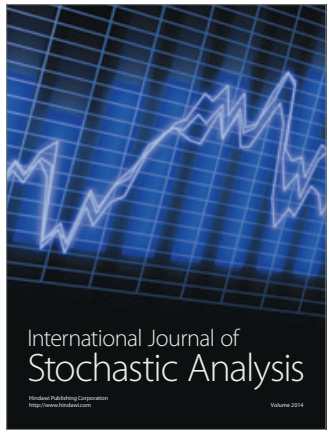

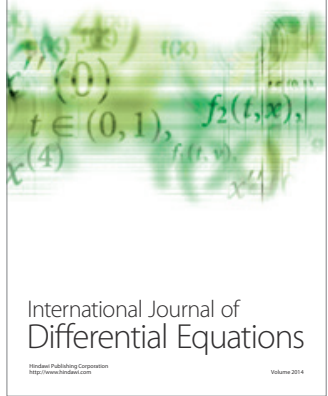
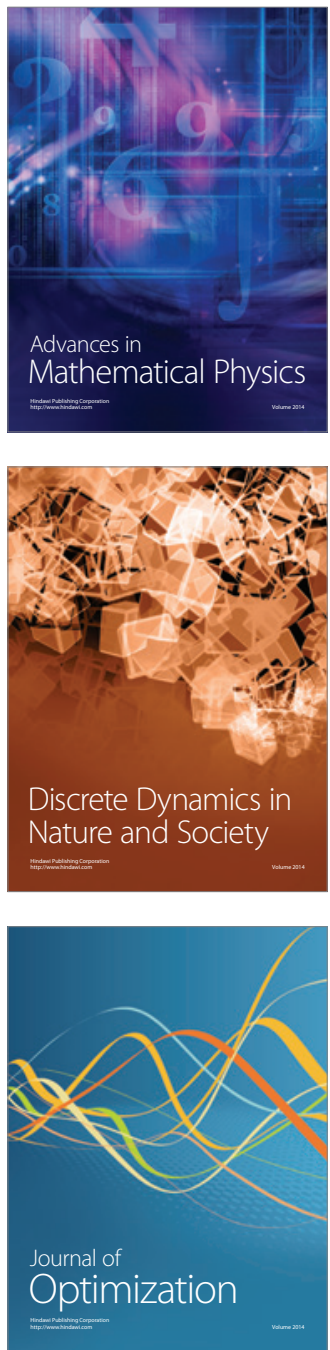